\title{
Mathematical Model for False Codling Moth Control Using Pheromone Traps
}

\author{
Jimrise Ochwach ${ }^{1 *}$, Mark O. Okongo ${ }^{2}$ and Moses M. Muraya ${ }^{3}$ \\ ${ }^{1}$ Department of Physical Sciences, Chuka University, P.O. Box 109-60400, Kenya. \\ ${ }^{2}$ Department of Physical Sciences, Chuka University, P.O. Box 109-60400, Kenya.r \\ ${ }^{3}$ Department of Plant Sciences, Chuka University, P.O. Box 109-60400, Kenya. \\ *ojimrise09@gmail.com:
}

\begin{abstract}
False codling moth (FCM) is regarded as the most significant indigenous pest. Over 70 host plants are attacked by larval, many of which are horticultural crops with fruit, pods, and berries, such as beans, grapes, citrus, capsicum, avocado, guava, pomegranate, and ornamental plants. They eat macadamia nuts, cotton, tea, and a variety of other wild plants as well. Female moths are drawn to the flower heads as well as other parts of the plant, making this pest especially problematic on roses grown for cut flowers. Therefore, controlling this pest is of importance. For more effective control, pheromone traps are used to capture males attracted to the artificial pheromone. In this study, mathematical model of FCM control using pheromone trap is developed. The model, is based on biological and ecological assumptions, and is governed by an ODE system. The coexistence and pest free equilibria is determined through a theoretical analysis. The theoretical analysis of the model allows for the identification of pheromone threshold values that are practical for field applications. We show that there is a threshold above which the global asymptotic stability of the trivial equilibrium is guaranteed. Finally, we demonstrate the theoretical results through numerical experiments.
\end{abstract}

Keywords: False codling moth, Mathematical modeling, stability Analysis, pest, Pheromone Traps, Plant pest model

\section{Introduction}

False codling moth (FCM), (Thaumatotibia leucotreta) is considered the most significant indigenous pest due to its potential economic impact on many horticultural and agricultural crops [22]. larval attack over 70 host plants, many of which are horticultural crops with fruit, pods, and berries, such as beans, grapes, citrus, capsicum, avocado, guava, pomegranate, and ornamental plants. They also feed on macadamia nuts, cotton, tea, and a variety of other wild plants. Female moths are attracted to lay their eggs on the flower heads as well as other parts of the plant, making this pest particularly problematic on roses grown for cut flowers [52].

Consequently, FCM is a major threat to food security, supply of raw material for manufacturing, foreign exchange and employment in many countries [10;11]. FCM is widely distributed across Africa and has been reported in over 40 Africa countries , including Kenya [42;49]. The FCM is not considered to be established outside of Africa [52]. However, it is commonly intercepted during quarantine inspections in Europe and United States [21].

Due to the presence of FCM, rose shipments from Kenya to Europe have been intercepted in recent years. If a single living FCM is discovered within a consignment at any stage of development, the entire consignment is rejected [26]. This is due to FCM being on the European Commission's (EC) list of harmful organisms that should be regulated as quarantine pests in order to prevent its introduction into Europe, where it could harm a variety of outdoor and greenhouse crops [36; 34]. As a result, because it is endemic to Sub-Saharan Africa, FCM is a phytosanitary concern and a barrier to export in most international markets $[25 ; 36]$.

On the other hand, according to European Union (EU) plant health (phytosanitary) regulations (EU 2016/2031), the false codling moth is now a quarantine pest on all crops. Special measures have been put in place for crops that are known entry points for serious pests that could harm Europe's agriculture or environment. These measures include stringent new requirements for rose exports in order to prevent the spread of FCM into the EU [26]. FCM interceptions on Kenyan roses have been extremely high (36 in 2018, 36 in 2019, and 24 up to June 2020). This high rate of interceptions has been attributed to increased inspection levels, which have increased from 5\% in 2011 to $10 \%$ now. As a 
result of the numbers observed in the previous three years, a drastic increase to $50 \%$ or even $100 \%$ checks for Kenyan roses is expected in 2021. This will be determined by the end of 2020 [26].

Planning efficient and cost-effective FCM control is a real challenge, which explains why most experimental FCM control strategies fail [4]. This is because certain parameters can be changed to make biological systems unstable or stable, i.e., if their values pass through bifurcation values. [38; 43; 22]. As a result, more scientific research on FCM interactions with hosts is required for effective pest management.

The demand for reliable pest infestation models is growing because they aid in defining problems, organizing thoughts, identifying areas to investigate, making predictions, generating hypotheses, and assisting in pest management decision-making. They also serve as standard comparisons and offer strategies to improve pest control decision-making [4;15]. Predicting population dynamics and evaluating pest control scenarios by agro-ecosystem under a variety of environmental conditions can reduce the number and cost of pest control interventions while improving crop yields and quality, as well as health and sustainability [55].

Most of the farmers rely on insecticide to reduce economic loss caused by FCM [28]. Chemical pesticides have been extensively used to control pest population such as FCM for a long time all over the world. However, their extensive use has led to environmental pollution and reduction of pest's natural enemies resulting to undesirable side effects on the environment [18]. Moreover, the development of insect resistance to the chemical products has also led to the rising need of stronger and more toxic pesticides to maintain their efficacy. Extensive use of pesticides is not a sustainable solution for pest control [4].

As a result, there is growing interest on development of control strategies that do not pollute the environment, these strategies have a special focus on the ecology and behaviour of the involved species. Therefore, to prevent devastating impact on economy [54], and ensure sufficient food security, social life, health and biodiversity; efficient control, understanding of biodiversity and management of FCM is essential [4; 45 ). Mating disruption is one such non polluting control strategies. It involves introducing artificial stimuli such as pheromones that interfere with normal mating $(8 ; 14 ; 4]$. In most insect species, such as moth, it is the females that emit the sex pheromone to attract males, from a distance of $40 \mathrm{~m}$ or more depending of the environmental conditions [18]. Pheromones are an appealing alternative to conventional pesticides and have shown promise as a component of integrated pest management. Pheromones play an important role in the lives of most insects, serving a variety of functions such as trail following, territorial behavior, dispersal, aggregation, and sexual activities, and they can also be used as alarm and defense chemicals. They are frequently highly specific to a specific species or species groups, and frequently consist of more than one specific chemical, making them highly selective. Since pheromone traps contain insecticide, once the males are attracted they are killed [24]. Thereby lowering the number of males available for mating.

Therefore, pheromone traps can play an important role in integrated pest management (IPM). Pheromone traps can be effective in disrupting mate location thus preventing mating and blocking reproduction cycle [37]. Subsequently, it leads to reduction of wild population of FCM in a farm [13]. In addition, the use of pheromones traps for pest monitoring and as a tool for accurately timing insecticide sprays has become a cornerstone feature of many prominent IPM programs [41]. However, pheromone lures reduces the need for application of chemical insecticide, and in some cases it is practiced as a stand-alone tactic. Today, mating disruption, as a technique is practiced worldwide for control of moth pests in fruits, vegetables, and forestry [16].

In a variety of contexts, pheromones have been proposed for use in pest control. One disadvantage of using pheromones for trapping is that they are typically only effective at low pest densities because high concentrations either confuse or repel the target species. [4; 7]. Some of the difficulties with pheromone trap method include the evaluation of orientation sites, the effective range of pheromone communication and dispersal, circadian rhythms in the release of female pheromones, the establishment of flight ranges, the variation in frequency of mating of males and females, the uneven distribution of breeding populations, and the interaction of pheromones with the various physical factors. Furthermore, some pest predators and parasites use pest pheromones as Kairomones to locate the pest. Thus, mass trapping for pests frequently attracts natural enemies of the pests, which could potentially undermine the control program [16].

Because of these difficulties, general mathematical modeling of pheromone trap control is more difficult and has a more limited applicability than if the biology of pheromone-induced behavior was simpler. However, one approach to dealing with some of these difficulties is to systematically change certain aspects of a model to account for the wide range of biological phenomena observed in nature [18]. Mathematical modeling is a useful tool for analyzing agricultural issues. Mathematical modeling applied to biological pest control problems allows for a qualitative and quantitative assessment of the pest's impact on natural enemy populations.

In this paper, we propose a simple mathematical model for false codling moth control using pheromone traps. In this model, the FCM is represented by the egg, the larval stages, the pupal stage, and adults stages and the susceptible host are represented by a system of ODE.

\section{Main Body}

\subsection{Model development}

In the absence of a pest, the susceptible $S_{h}$ host grows logistically to maturity at the rate $\alpha S_{h}(t)\left(1-\frac{S_{h}(t)}{K}\right)$, where $\alpha$ represents the intrinsic growth rate and $K$ represents the environmental carrying capacity. Hosts can only be infected by the FCM after a fertilized fertile female $\left(F_{f f}\right)$ deposits eggs, which hatch into larvae and burrow into the host's rind [44]. Once infected by the larvae $\left(L_{f}\right)$, the host fruit never recovers and produces no yield [2], and it must be removed from the farm or fall to the ground. The host invasion is assumed to follow the Holling-type II functional response $\frac{\xi S_{h}(t) L_{f}(t)}{m+S_{h}(t)}$, where $\xi$ is the host invasion rate by the larvae and $m$ is the half saturation constant [46]. When a healthy host reaches maturity, it is harvested at the rate of $\mu_{1}$. The equation that governs the susceptible host compartment is given 
by equation 1 based on the above assumptions:

$\frac{d S_{h}(t)}{d t}=\alpha\left(1-\frac{S_{h}(t)}{K_{h}}\right) S_{h}(t)-\frac{\xi S_{h}(t) L_{f}(t)}{m+S_{h}(t)}-\mu_{1} S_{h}(t)$

It is important to note that the FCM population is made up of Eggs $\left(E_{f}\right)$, Larvae $\left(L_{f}\right)$, Pupae $\left(P_{f}\right)$, and Adults (Fertile female $\left(F_{f}\right)$, Fertile male $\left(M_{f}\right)$, and Fertilized fertile female $\left(F_{f f}\right)$. Each developmental stage is affected by temperature and food availability [44]. The oviposition rate $r F f f$, where $r$ is the intrinsic egg laying rate and $F_{f f}$ is the number of fertilized fertile females of FCM, determines the number of eggs produced. Because eggs are limited by the host's carrying capacity, the effective egg production rate is given by $r F_{f f}\left(1-\frac{E_{f}(t)}{A}\right) \phi S_{h}(t)$, where $\left(1-\frac{E_{f}(t)}{A}\right) \phi S_{h}(t)$ is the host's available capacity to receive eggs from fertilized fertile female, $A$ is the host's carrying capacity, and $\phi$ is the eggs conversion rate. If the transfer rate of egg to larvae is $\lambda_{1}$ and $\omega_{1}$ is natural mortality rate of egg stage at temperature $\tau_{1}$ then, the general equation that describes the egg stage is given by equation 2 :

$\frac{d E_{f}(t)}{d t}=r F_{f f}(t)\left(1-\frac{E_{f}(t)}{A}\right) S_{h}(t)-\left(\lambda_{1}+\tau_{1} \omega_{1}\right) E_{f}(t)$

The larval stage of FCM is the most damaging because larvae can burrow into the fruit and cause damage inside the fruit while displaying few symptoms on the fruit. Secondary pests such as fungal organisms and scavengers can become vulnerable to damaged fruit [12]. If $\omega_{2}$ is the natural mortality rate of larvae at temperature $\tau_{2}$ and $\lambda_{2}$ is the rate at which larvae pupate. Then, using $a$ to represent the larvae's conversion efficiency, we can calculate the contribution of the larval population from susceptible hosts. Then, equation 3 describes the process that describes the fertile larval stage compartment:

$\frac{d L_{f}(t)}{d t}=\lambda_{1} E_{f}(t)+\frac{a \xi S_{h}(t) L_{f}(t)}{m+S_{h}(t)}-\left(\lambda_{2}+\tau_{2} \omega_{2}\right) L_{f}(t)$

When the final instar larva is ready to pupate, it falls to the ground, spins a cocoon, and pupates in the soil's top layer [12]. It becomes inactive at the early pupal stage and can take longer to develop depending on the temperature and humidity of the environment [45]. Male and female pupae can be distinguished after maturation at the pupal stage[10;45]. Because the transfer rate from larval to pupal stage is $\lambda_{2}$, we can calculate the mortality rate at the pupal stage at temperature $\tau_{3}$ and the transfer rate from pupal to adult FCM by letting $\omega_{3}$ be the mortality rate at the pupal stage and $\lambda_{3}$ be the transfer rate from pupal to adult FCM. Then, equation 4 gives the equations that describe the pupal stage:

$\frac{d P_{f}(t)}{d t}=\lambda_{2} L_{f}(t)-\left(\omega_{3}+\tau_{3} \lambda_{3}\right) P_{f}(t)$

After emerging from the pupal stage, it is assumed that the fertile female FCM can successfully mate with the fertile male FCM in order to move to the fertilized fertile female compartment at a rate of $\lambda_{4}$, and that after mating, the fertilized fertile female returns to the fertile female FCM compartment at a rate of $\delta_{1}$ in order to mate again. Since the transfer rate from larval to pupal stage is $\lambda_{3}$, and assuming that the natural mortality of fertile females is $\omega_{4}$ at temperature $\tau 4$, and letting $\kappa$ be the fraction of pupal population that moves to fertile female stage $\left(F_{f}\right)$. Then, as shown in equation 5 , the equation that describes the fertile female compartment is modeled:

$\frac{d F_{f}(t)}{d t}=\kappa \lambda_{3} P_{f}(t)+\delta_{1} F_{f f}(t)-\left[\lambda_{4}+\tau_{4} \omega_{4}\right] F_{f}(t)$

If the pupal stage to fertile male FCM transfer rate is $\lambda_{4}$ and $(1-\kappa)$ is the fraction of the pupal population that moves to the fertile male stage. Assuming that the natural mortality of the fertile male FCM at temperature $\tau_{5}$ is given by $\omega_{5}$ and that there is no further transfer in the fertile male compartment and that the only population reduction of fertile male is due to natural death. Then, equation 6 describes the equation that governs the adult male compartment:

$\frac{d M_{f}(t)}{d t}=(1-\kappa) \lambda_{3} P_{f}(t)-\tau_{5} \omega_{5} M_{f}(t)$

For mating to occur, the female FCM calls the male FCM via pheromone release that begins several hours after dark [45], and the sex pheromone assists the male FCM in allocating themselves in space and time for the available mate [55]. Because FCM mating is a complex process, it is assumed that male FCM can mate several times per day and throughout its life. After laying fertile eggs that hatch and burrow into the host fruit, the fertilized fertile female is usually the one responsible for causing direct damage to the host. It is also assumed that in order to enter the fertilized fertile female FCM compartment, fertile female FCM must mate with a fertile male FCM. If $\lambda_{4}$ is the number of female FCM that move to the fertilized fertile female FCM compartment, $\delta_{1}$ is the number of fertilized fertile females that return to the fertile female compartment for mating, and $\omega_{6}$ is the natural mortality rate of fertilized fertile females at temperature $\tau_{6}$. Then, equation 7 is given to describe the rate of change at the fertilized fertile female FCM compartment:

$\frac{d F_{f f}(t)}{d t}=\lambda_{4} F_{f}(t)-\left(\delta_{1}+\tau_{6} \omega_{6}\right) F_{f f}(t)$

In order to incorporate the pheromone lures in the model, we follow the method proposed by Barclay and Van den Driessche, [7] and Anguelove et al.[4]. Let $F_{p}$ be the number of pheromone lure released into the population at a rate $v$ and assuming that pheromone undergo decay at the rate $\mu$, then the equation that governs pheromone movement can be given by equation 8 : 
$\frac{d F_{p}(t)}{d t}=v-\mu F_{p}(t)$

Assume that the pheromone concentration is equal to the number of fertile female FCM, resulting in $\left(F_{p}+F_{f}\right)$ as the total number of female moths in the population. As a result, the probability that a fertile male will be attracted to a fertile female is $\frac{F_{f}}{F_{f}+F_{f}}$, whereas the probability that a fertile male will be attracted to pheromone traps is $\frac{F_{f}}{F_{p}+F_{f}}$. Then, equation 9 , describes the evolution of fertile male compartment in the presence of pheromone lure:

$$
\frac{d M_{f}(t)}{d t}=(1-\kappa) \lambda_{3} P_{f}(t)-\left[\left(\frac{F_{P}}{F_{f}+F_{p}}\right)+\tau_{5} \omega_{5}\right] M_{f}(t)
$$

\subsection{Model Flow Chart}

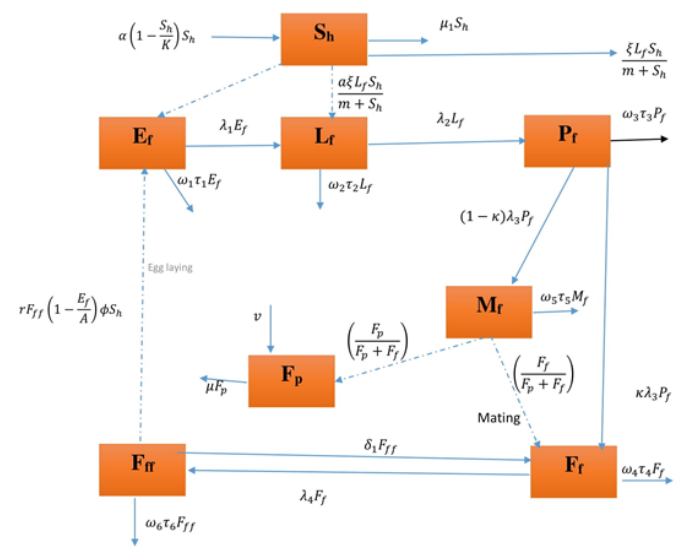

Figure 1: Model Flow Chart

\subsection{Model Equation}

Taking into account the above considerations, we have the schematic flow diagram shown in Figure 1 (Model Flow Chart). From figure 1, the model will be governed by the following system of equations 10 :

$$
\begin{aligned}
\frac{d S_{h}(t)}{d t} & =\alpha\left(1-\frac{S_{h}(t)}{K_{h}}\right) S_{h}(t)-\frac{\xi S_{h}(t) L_{f}(t)}{m+S_{h}(t)}-\mu_{1} S_{h}(t) \\
\frac{d E_{f}(t)}{d t} & =r F_{f f}(t)\left(1-\frac{E_{f}(t)}{A}\right) S_{h}(t)-\left(\lambda_{1}+\tau_{1} \omega_{1}\right) E_{f}(t) \\
\frac{d L_{f}(t)}{d t} & =\lambda_{1} E_{f}(t)+\frac{a \xi S_{h}(t) L_{f}(t)}{m+S_{h}(t)}-\left(\lambda_{2}+\tau_{2} \omega_{2}\right) L_{f}(t) \\
\frac{d P_{f}(t)}{d t} & =\lambda_{2} L_{f}(t)-\left(\omega_{3}+\tau_{3} \lambda_{3}\right) P_{f}(t) \\
\frac{d F_{f}(t)}{d t} & =\kappa \lambda_{3} P_{f}(t)+\delta_{1} F_{f f}(t)-\left[\lambda_{4}+\tau_{4} \omega_{4}\right] F_{f}(t) \\
\frac{d M_{f}(t)}{d t} & =(1-\kappa) \lambda_{3} P_{f}(t)-\left[\left(\frac{F_{P}}{F_{f}+F_{p}}\right)+\tau_{5} \omega_{5}\right] M_{f}(t) \\
\frac{d F_{f f}(t)}{d t} & =\lambda_{4} F_{f}(t)-\left(\delta_{1}+\tau_{6} \omega_{6}\right) F_{f f}(t) \\
\frac{d F_{p}(t)}{d t} & =v-\mu F_{p}(t)
\end{aligned}
$$

\subsection{Model Parameter Values}

The parameter values used in this study were obtained from literature, or assumed after making reasonable biological and mathematical assumption. 


\begin{tabular}{|l|l|l|l|}
\hline Symbol & Parameter & Value & Source \\
\hline$\alpha$ & Intrinsic growth rate of the host & $2.76 \mathrm{~mm}$ per Month & {$[53]$} \\
$K_{h}$ & Environmental carrying capacity & $1000-1500$ fruits per tree & Assumed \\
$A$ & Fruit carrying capacity of eggs & $3-8$ eggs per fruit & {$[34]$} \\
$\xi$ & Infestation rate of the pest & $0.417-0.5417$ & {$[34]$} \\
$\lambda_{1}$ & Transfer rate of $J$ to adult FCM & $0.06-0.217$ & {$[41]$} \\
$\lambda_{2}$ & Transfer rate from L stage to $P_{m}$ & $0.01031-0.05025$ & {$[41]$} \\
$\omega_{1}$ & Mortality rate of fertile E & 0.03 & {$[41]$} \\
$\omega_{2}$ & Mortality rate of fertile L & $0.009-0.115$ & {$[41]$} \\
$\omega_{3}$ & Mortality rate of fertile Pupa & 0.007 & {$[41]$} \\
$\mu_{1}$ & Harvesting rate of the host & 0.06 & Assumed \\
$r$ & Intrinsic egg laying rate & $4.725 \mathrm{day}^{-1}$ female $^{-1}$ & {$[41]$} \\
$m$ & Half saturation constant & 0.8 & {$[7]$} \\
$v$ & Pheromone concentration & $10 \mathrm{ngm}^{-3}$ & {$[29]$} \\
$\mu$ & Pheromone decay rate & 0.8 & {$[29]$} \\
\hline
\end{tabular}

Table 1: Parameter values of FCM

\subsection{Boundedness of the Solution}

The time evolution of the individuals of each class is revealed by the solutions of the model system 10 . For any dynamical system it is important to ensure that the system is well-posed so that the solution reflect the natural phenomena [11]. Therefore, for the solutions of the model system 10 to be mathematically and epidiomologically meaningful, its important to prove that all its state variables are positive and bounded for all time $t$. That is, the solution of the system with positive initial value will remain positive for all time $t \geq 0$. This can be established using theorem 1:

Theorem 1. If the initial conditions of the model system 10 are in domain $\mathscr{D}$ such that:

$$
\begin{aligned}
\mathscr{D} & =\left\{\left(S_{h}(t), E_{f}(t), L_{f}(t), P_{f}(t), F_{f}(t), M_{f}(t), F_{f f}(t), F_{p}(t)\right)\right. \\
& \left.\in \Re_{+}^{8}: N(t) \leq \frac{\hat{k}}{q}(\alpha+1)+\varepsilon\right\}
\end{aligned}
$$

Then all solutions of the system equation 10 enter and remain in $\mathscr{D}$

Proof. Consider the set $S_{h}(t), E_{f}(t), L_{f}(t), P_{f}(t), F_{f}(t), M_{f}(t), F_{f f}(t), F_{p}(t)$ with any solution of the system model 10 , such that the total population expressed as $N(t)=S_{h}(t)+E_{f}(t)+L_{f}(t)+P_{f}(t)+F_{f}(t)+M_{f}(t)+F_{f f}(t)+F_{p}(t)$ and $\frac{d N(t)}{d t}=\frac{d S_{h}}{d t}+\frac{d E_{f}}{d t}+\frac{d L_{f}}{d t}+\frac{d P_{f}}{d t}+\frac{d F_{f}}{d t}+$ $\frac{d M_{f}}{d t}+\frac{d F_{f f}}{d t}+\frac{d F_{p}}{d t}$. Now using the foregoing expression in the addition of the system model 11, yields:

$\frac{d N(t)}{d t}=\frac{d}{d t}\left\{S_{h}(t)+E_{f}(t)+L_{f}(t)+P_{f}(t)+F_{f}(t)+M_{f}(t)+F_{f f}(t)+F_{p}(t)\right\}$

$$
\begin{aligned}
\frac{d N(t)}{d t} & =\frac{d S_{h}}{d t}+\frac{d E_{f}}{d t}+\frac{d L_{f}}{d t}+\frac{d P_{f}}{d t}+\frac{d F_{f}}{d t}+\frac{d M_{f}}{d t}+\frac{d F_{f f}}{d t}+\frac{d F_{p}}{d t} \\
& +\left[\alpha\left(1-\frac{S_{h}(t)}{K_{h}}\right) S_{h}(t)-\frac{\xi S_{h}(t) L_{f}(t)}{m+S_{h}(t)}-\mu_{1} S_{h}(t)\right] \\
& +\left[r F_{f f}(t)\left(1-\frac{E_{f}(t)}{A}\right) S_{h}(t)-\left(\lambda_{1}+\tau_{1} \omega_{1}\right) E_{f}(t)\right] \\
& +\left[\lambda_{1} E_{f}(t)+\frac{a \xi S_{h}(t) L_{f}(t)}{m+S_{h}(t)}-\left(\lambda_{2}+\tau_{2} \omega_{2}\right) L_{f}(t)\right] \\
& +\left[\lambda_{2} L_{f}(t)-\left(\lambda_{3}+\tau_{3} \omega_{3}\right) P_{f}(t)\right] \\
& +\left[\kappa \lambda_{3} P_{f}(t)+\delta_{1} F_{f f}(t)-\left[\lambda_{4}+\tau_{4} \omega_{4}\right] F_{f}(t)\right] \\
& +\left[(1-\kappa) \lambda_{3} P_{f}(t)-\left[\left(\frac{F_{P}}{F_{f}+F_{p}}\right)+\tau_{5} \omega_{5}\right] M_{f}(t)\right] \\
& +\left[\lambda_{4} F_{f}(t)-\left(\delta_{1}+\tau_{6} \omega_{6}\right) F_{f f}(t)\right] \\
& +v-\mu F_{p}(t)
\end{aligned}
$$

Which simplifies to:

$$
\begin{aligned}
\frac{d N(t)}{d t} & \leq \alpha\left(1-\frac{S_{h}(t)}{K_{h}}\right) S_{h}(t)+r F_{f f}(t)\left(1-\frac{E_{f}(t)}{A}\right) S_{h}(t)+\frac{\xi S_{h}(t) L_{f}(t)}{m+S_{h}(t)}(a-1) \\
& +\lambda_{4} F_{f}(t)+v-\left(\frac{F_{p}(t)}{F_{f}(t)+F_{p}(t)}\right) \\
& -\left\{\mu_{1} S_{h}(t)+\tau_{1} \omega_{1} E_{f}+\tau_{2} \omega_{2} L_{f}+\tau_{3} \omega_{3} P_{f}+\tau_{4} \omega_{4} F_{f}+\tau_{5} \omega_{5} M_{f}+\tau_{6} \omega_{6} F_{f f}+v F_{p}\right\}
\end{aligned}
$$


Where $\hat{k}=\max \{S(0), K\}$ and $\Psi=\min \left\{1, \tau_{1} \omega_{1}, \tau_{2} \omega_{2}, \tau_{3} \omega_{3}, \tau_{4} \omega_{4}, \tau_{5} \omega_{5}, \tau_{6} \omega_{6} v\right\}$ Then

$\frac{d N}{d t}+\Psi N=\hat{k}(\alpha+1)$

and its solutions become:

$N(t) \leq \frac{\hat{k}}{\Psi}(\alpha+1)(1-\exp (-\Psi t))+N(0) \exp (-\Psi t)$

At $t \rightarrow \infty$, we have $N(t) \leq \frac{\hat{k}}{\Psi}(\alpha+1)$. Implies that the solution is bounded for $0 \leq N \leq \stackrel{\hat{k}}{\Psi}(\alpha+1)$. Thus all solutions of the model system 10 in $\mathfrak{R}_{+}^{8}$ are restricted in the region: $\mathscr{D}=\left\{\left(S_{h}(t), E_{f}(t), L_{f}(t), P_{f}(t), F_{f}(t), M_{f}(t), F_{f f}(t), F_{p}(t)\right) \in \mathfrak{R}_{+}^{8}: N(t) \leq \frac{\hat{k}}{q}(\alpha+1)+\varepsilon\right\}$ for all $\varepsilon>0$ and $t \rightarrow 0$. Therefore, the feasible solutions set for the host pest interaction model is positively invariant, biologically meaningful and mathematically well-posed.

\subsection{Equilibria Analysis}

In this study, we look at three different types of equilibrium: pest-free equilibrium $\left(E_{0}\right)$, coexistence equilibrium $\left(E_{1}\right)$, and host-free equilibrium $\left(E_{2}\right)$.

\subsubsection{Pest Free Equilibrium Point}

It is assumed that there is no pest prevalence in the system at the pest-free equilibrium points $\left(E_{0}\right)$, so the population of the host grows logistically to maturity until harvesting time. To analyze pest-free equilibrium point (PFE), the system model 10 pest components are set to zero, and because there is no pest prevalence, the pheromone compartment is also set to zero, resulting in: $E_{f} *=0, L_{f} *=0, P_{f} *=0, F_{f} *=$ $0, M_{f} *=0, F_{f f^{*}}=0, F_{p^{*}}=0$. This is accomplished by zeroing out the right-hand sides of the model system 10 and solved. We now solve the first equation of the system model 10 which reduces to equation 13 :

$0=\alpha\left(1-\frac{S_{h}^{*}(t)}{K_{h}}\right) S_{h}^{*}(t)-\frac{\xi S_{h}^{*}(t) L_{f}^{*}(t)}{m+S_{h}^{*}(t)}-\mu_{1} S_{h}^{*}(t)$

Since $L_{f}^{*}=0$ and $F_{f}^{*}=0$. Then, the solutions of $S_{h}^{*}$ in equation 13 is described by equation 14 :

$S_{h}^{*}=0$

$S_{h}^{*}=K_{h}\left(\alpha-\mu_{1}\right)$

It is important to note that when $S_{h}^{*}(t)=0$, then we have a trivial solution. Therefore we settle for the second solution $S_{h}^{*}=K_{h}\left(\alpha-\mu_{1}\right)$ to represent the solution of $S_{h}^{*}$ in the absence of pest. Consequently, the pest free equilibrium points is given by equation 15:

$E_{0}=\left(K_{h}\left(\alpha-\mu_{1}\right), 0,0,0,0,0,0,0\right)$

\subsection{Host Free Equilibrium Points}

At this equilibrium point $E_{1}$, it is assumed that that the host is absent with the existence FCM pest in the presence of pheromone traps and sterile insect such that, $S_{h}^{\&}=0, E_{f}^{\&} \neq 0, L_{f}^{\&} \neq 0, P_{f}^{\&} \neq 0, F_{f}^{\&} \neq 0, M_{f}^{\&} \neq 0, F_{f f}^{\&} \neq 0, F_{p}^{\&} \neq 0$.

$$
\begin{aligned}
0 & =\left(\lambda_{1}+\tau_{1} \omega_{1}\right) E_{f}^{\&}(t) \\
0 & =\lambda_{1} E_{f}^{\&}(t)-\left(\lambda_{2}+\tau_{2} \omega_{2}\right) L_{f}^{\&}(t) \\
0 & =\lambda_{2} L_{f}^{\&}(t)-\left(\lambda_{3}+\tau_{3} \omega_{3}\right) P_{f}^{\&}(t) \\
0 & =\kappa \lambda_{3} P_{f}^{\&}(t)+\delta_{1} F_{f f}^{\&}(t)-\left[\lambda_{4}+\tau_{4} \omega_{4}\right] F_{f}^{\&}(t) \\
0 & =(1-\kappa) \lambda_{3} P_{f}^{\&}(t)-\left[\left(\frac{F_{P}^{\&}}{F_{f}^{\&}+F_{p}^{\&}}\right)+\tau_{5} \omega_{5}\right] M_{f}^{\&}(t) \\
0 & =\lambda_{4} F_{f}^{\&}(t)-\left(\delta_{1}+\tau_{6} \omega_{6}(t)\right) F_{f f}^{\&}(t) \\
0=v & -\mu F_{p}^{\&}(t)
\end{aligned}
$$

Since $S_{h}^{\&}=0$, it is easy to see that $E_{f}^{\&}=L_{f}^{\&}=P_{f}^{\&}=F_{f}^{\&}, M_{f}^{\&}=F_{f f}^{\&}=0$,

Therefore, the host free equilibrium can be given by equation 17 :

$E_{1}=\left(0,0,0,0,0,0,0, \frac{v}{\mu}\right)$

Equation 17 shows that at host-free equilibrium, the pest population is zero. The numerical simulation illustrated in Figure 2 shows that the total population of FCM reduces to zero regardless of the initial FCM population, indicating that the population of FCM becomes extinct because their survival is dependent on the availability of the susceptible host. 


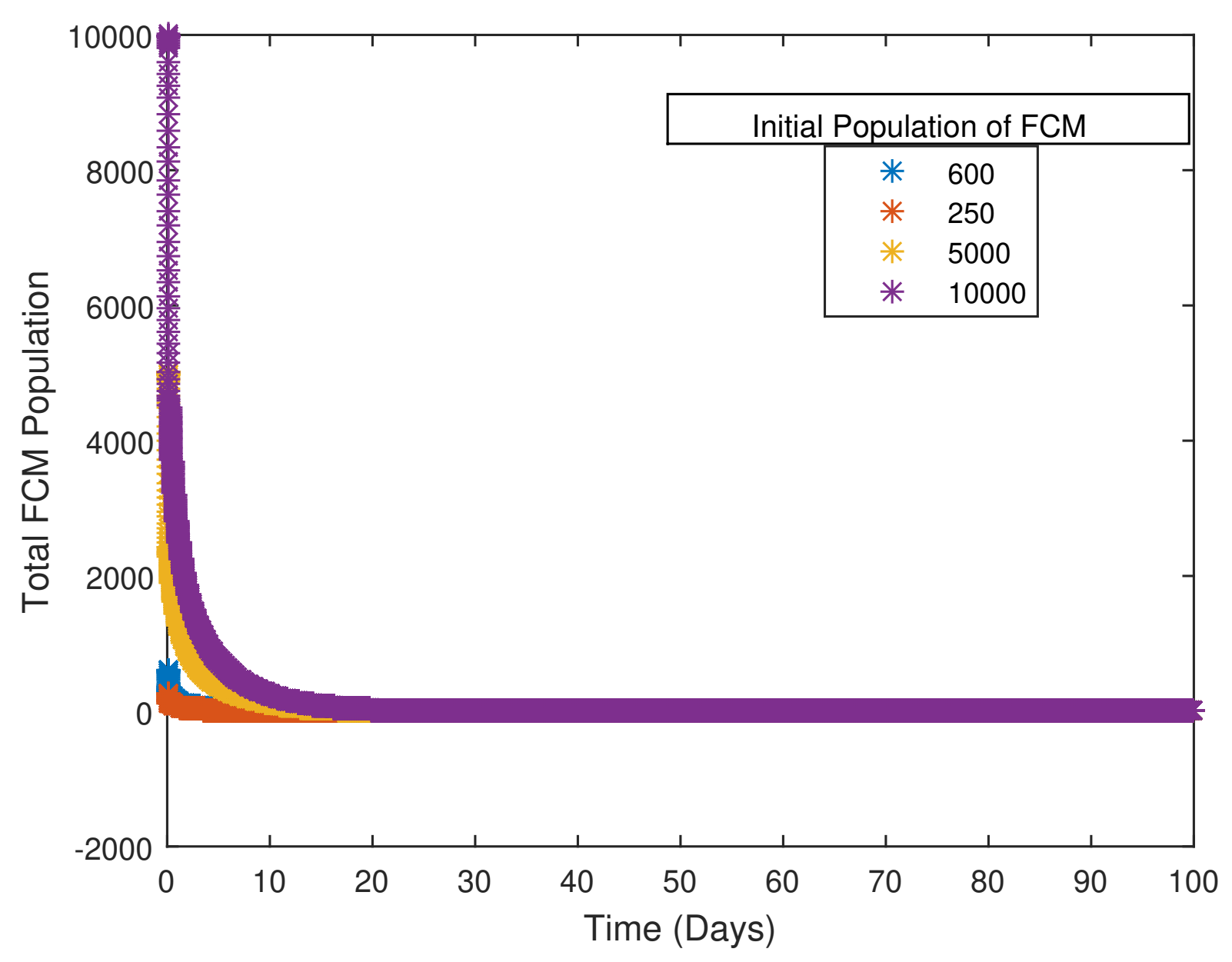

Figure 2: FCM Population against Time at Host Free Equilibrium

The host free equilibrium point is considered trivial in this study, so it is not subjected to stability analysis.

\subsubsection{Coexistence Equilibrium Points}

In the presence of FCM, host and pheromone traps, we assume that $S_{h} \geq 0, E_{f} \geq 0, L_{f} \geq 0, P_{f} \geq 0, F_{f} \geq 0, M_{f} \geq 0, F_{f f} \geq 0, F_{p} \geq 0$. The system model has an equilibrium point called coexistence equilibrium point denoted by $E_{2}=\left(S_{h}^{* \&}, E_{f}^{* \&}, L_{f}^{* \&}, P_{f}^{* \&}, F_{f}^{* \&}, M_{f}^{* \&}, F_{f f}^{* \&}, F_{p}^{* \&} \geq 0\right.$. $E_{2}$ is the steady-state solution in which FCM coexist naturally in a susceptible host population. This is accomplished by zeroing out the right-hand sides of the host pest interaction model 10 equations as shown in equation 18 , and solving for the values of $S_{h}^{* \&}, E_{f}^{* \&}, L_{f}^{* \&}, P_{f}^{* \&}$, $F_{f}^{* \&}, M_{f}^{* \&}, F_{f f}^{* \&}, F_{p}^{* \&} \geq 0$.

$$
\begin{aligned}
0 & =\alpha\left(1-\frac{S_{h}^{* \&}(t)}{K_{h}}\right) S_{h}^{* \&}(t)-\frac{\xi S_{h}^{* \&}(t) L_{f}^{* \&}(t)}{m+S_{h}^{* \&}(t)}-\mu_{1} S_{h}^{* \&}(t) \\
0 & =r F_{f f}^{* \&}(t)\left(1-\frac{E_{f}^{* \&}(t)}{A}\right) S_{h}^{* \&}(t)-\left(\lambda_{1}+\tau_{1} \omega_{1}\right) E_{f}^{* \&}(t) \\
0 & =\lambda_{1} E_{f}^{* \&}(t)+\frac{a \xi S_{h}^{* \&}(t) L_{f}^{* \&}(t)}{m+S_{h}^{* \&}(t)}-\left(\lambda_{2}+\tau_{2} \omega_{2}\right) L_{f}^{* \&}(t) \\
0 & =\lambda_{2} L_{f}^{* \&}(t)-\left(\lambda_{3}+\tau_{3} \omega_{3}\right) P_{f}^{* \&}(t) \\
0 & =\kappa \lambda_{3} P_{f}^{* \&}(t)+\delta_{1} F_{f f}^{* \&}(t)-\left[\lambda_{4}+\tau_{4} \omega_{4}\right] F_{f}^{* \&}(t) \\
0 & =(1-\kappa) \lambda_{3} P_{f}^{* \&}(t)-\tau_{5} \omega_{5} M_{f}^{* \&}(t) \\
0 & =\lambda_{4} F_{f}^{* \&}(t)-\left(\delta_{1}+\tau_{6} \omega_{6}(t)\right) F_{f f}^{* \&}(t) \\
0= & -\mu F_{p}^{* \&}(t)
\end{aligned}
$$


Solving first equation of the equation system 18, we proceed as shown in equation 19 :

$$
\begin{array}{r}
\alpha\left(1-\frac{S_{h}^{* \&}(t)}{K_{h}}\right) S_{h}^{* \&}(t)-\frac{\xi S_{h}^{* \&}(t) L_{f}^{* \&}(t)}{m+S_{h}^{* \&}(t)}-\mu_{1} S_{h}^{* \&}(t)=0 \\
\alpha\left(1-\frac{S_{h}^{* \&}(t)}{K_{h}}\right)-\frac{\xi L_{f}^{* \&}(t)}{m+S_{h}^{* \&}(t)}-\mu_{1}=0 \\
\alpha\left(1-\frac{S_{h}^{* \&}(t)}{K_{h}}\right)\left(m+S_{h}^{* \&}(t)\right)-\xi L_{f}^{* \&}-\mu_{1}\left(m+S_{h}^{* \&}(t)\right)=0 \\
\left(S_{h}^{* \&}\right)^{2}+\left(\mu_{1}+\frac{m}{K_{h}}-\alpha\right) K_{h} S_{h}^{* \&}+\left(\xi L_{f}^{* \&}+\mu m-\alpha m\right) K_{h}=0
\end{array}
$$

Therefore, the solution of $S_{h}^{* \&}(t)$ is given in equation 20:

$S_{h}^{* \&}(t)=-\frac{1}{2}\left[\left(\mu_{1}+\frac{m}{K_{h}}-\alpha\right) K_{h} \pm \sqrt{\left(\mu_{1}+\frac{m}{K_{h}}-\alpha\right)-4\left(\xi_{\left.L_{f}^{* \&}+\mu m-\alpha m\right) K_{h}}\right.}\right]$

Similarly, solving the second equation of equation 18 , we proceed as shown in equation 21 :

$$
\begin{array}{r}
r F_{f f}^{* \&}(t)\left(1-\frac{E_{f}^{* \&}(t)}{A}\right) S_{h}^{* \&}(t)-\left(\lambda_{1}+\tau_{1} \omega_{1}\right) E_{f}^{* \&}(t)=0 \\
r F_{f f}^{* \&}(t) S_{h}^{* \&}(t)-\frac{r F_{f f}^{* \&}(t) S_{h}^{* \&}(t)}{A} E_{f}^{* \&}(t)-\left(\lambda_{1}+\tau_{1} \omega_{1}\right) E_{f}^{* \&}(t)=0
\end{array}
$$

Therefore, the solution of $E_{f}^{* \&}(t)$ is given in equation 22 :

$E_{f}^{* \&}(t)=\frac{A r F_{f f}^{* \&}(t) S_{h}^{* \&}(t)}{r F_{f f}^{* \&}(t) S_{h}^{* \&}(t)+A\left(\lambda_{1}+\tau_{1} \omega_{1}\right)}$

Similar procedure can be followed to get the solutions of $L_{f}^{* \&}, P_{f}^{* \&}, F_{f}^{* \&}, M_{f}^{* \&}, F_{f f}^{* \&}, F_{p}^{* \&}$ as given in equation 23 :

$$
\begin{aligned}
L_{f}^{* \&} & =\frac{\lambda_{1} E_{f}^{* \&}}{\left(\lambda_{2}+\tau_{2} \omega_{2}\right)\left(\left(\lambda_{2}+\tau_{2} \omega_{2}\right)-\frac{a \xi S_{h}^{* \&}}{m+S_{h}^{* \&}}\right)} \\
P_{f}^{* \&} & =\frac{\lambda_{2} L_{f}^{* \&}}{\left(\lambda_{3}+\tau_{3} \omega_{3}\right)} \\
F_{f}^{* \&} & =\frac{\kappa \lambda_{3} P_{f}^{* \&}+\delta_{1} F_{f f}^{* \&}}{\tau_{4} \omega_{4}+\lambda_{4}} \\
M_{f}^{* \&} & =\frac{\frac{\lambda_{4}+\tau_{4} \omega_{4}-\delta_{1}}{\kappa \lambda_{3}}}{\left(\frac{\frac{u}{v}}{F_{f}^{* \&}+\frac{u}{v}}+\tau_{5} \omega_{5}\right)} \\
F_{f f}^{* \&} & =\frac{\lambda_{4} M_{f}^{* \&} F_{f}^{* \&}}{\left(\delta_{1}+\tau_{6} \omega_{6}\right)} \\
F_{p}^{* \&} & =\frac{u}{\mu}
\end{aligned}
$$

The analytical solution to equation 23 is difficult to find. As a result, we employ numerical solutions from the MATLAB software, as shown in Figures 3 . The figure depicts the total pest population versus time in the absence of the control measure at the coexistence equilibrium point using the parameter values from Table 1. 


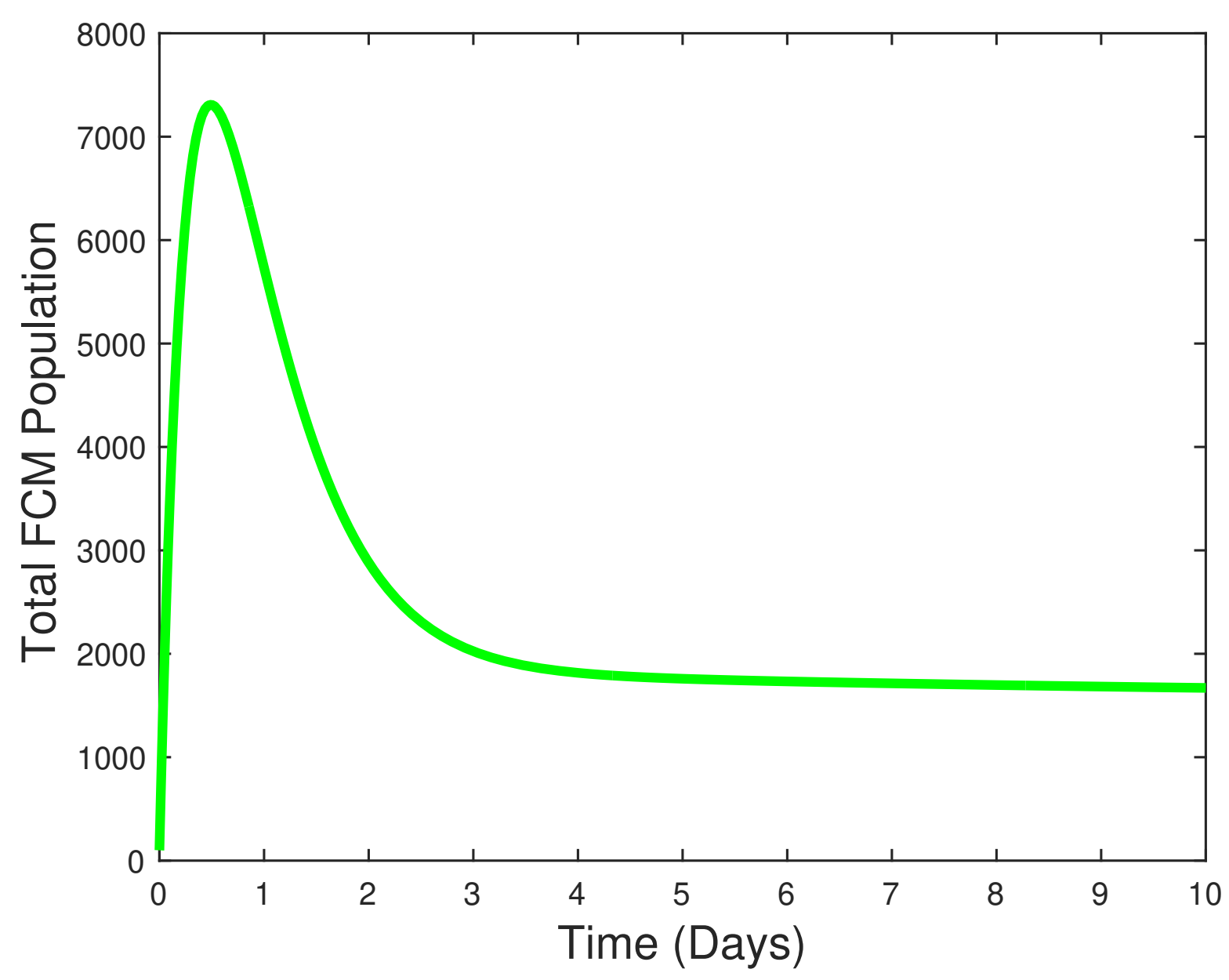

Figure 3: Total FCM Population against Time at Coexistence Equilibrium

\section{Basic Reproduction Number}

We calculate the basic reproduction number in this study using the method proposed by Anguelove et al. [4] and Lui and Dai [31]. Assuming the FCM is present in the host population and that the larval stage is the attacking stage. The basic reproduction number for the model system 10 can be computed using the van den Driessch and Watmough method ([4;38; 49]). In this case, we consider FCM compartments that are in charge of offspring reproduction; in this case, the system model 10 is reordered and reduces to equation 24:

$$
\begin{aligned}
& \frac{d L_{f}(t)}{d t}=\lambda_{1} E_{f}(t)+\frac{a \xi S_{h}(t) L_{f}(t)}{m+S_{h}(t)}-\left(\lambda_{2}+\tau_{2} \omega_{2}\right) L_{f}(t) \\
& \frac{d P_{f}(t)}{d t}=\lambda_{2} L_{f}(t)-\left(\lambda_{3}+\tau_{3} \omega_{3}\right) P_{f}(t) \\
& \frac{d F_{f}(t)}{d t}=\kappa \lambda_{3} P_{f}(t)+\delta_{1} F_{f f}(t)-\left[\lambda_{4}+\tau_{4} \omega_{4}\right] F_{f}(t) \\
& \frac{d M_{f}(t)}{d t}=(1-\kappa) \lambda_{3} P_{f}(t)-\left[\left(\frac{F_{P}}{F_{f}+F_{p}}\right)+\tau_{5} \omega_{5}\right] M_{f}(t) \\
& \frac{d F_{f f}(t)}{d t}=\lambda_{4} F_{f}(t)-\left(\delta_{1}+\tau_{6} \omega_{6}(t)\right) F_{f f}(t) \\
& \frac{d E_{f}(t)}{d t}=r F_{f f}(t)\left(1-\frac{E_{f}(t)}{A}\right) \phi S_{h}(t)-\left(\lambda_{1}+\tau_{1} \omega_{1}\right) E_{f}(t) \\
& \frac{d F_{p}(t)}{d t}=v-\mu F_{p}(t)
\end{aligned}
$$

Let $\mathscr{F}_{i}(y)$ be recruitment rate of new individuals in compartment $i, \mathscr{V}_{i}^{-}(y)$ the transfer of individuals out of the compartment $i$ and $\mathscr{V}_{i}^{+}(y)$ the transfer of individuals into the compartment $i$. Then, the system model 24 can be rewritten as shown in equation 25:

$\frac{d y}{d t}=\mathscr{F}_{i}(y)-\mathscr{Y}_{i}(y)$ 
Where $i=1, \ldots 6$ and $\mathscr{V}_{i}(y)=\mathscr{V}_{i}^{-}(y)-\mathscr{V}_{i}^{+}(y)$. With $\mathscr{F}_{i}(y)$ and $\mathscr{V}_{i}(y)$ forms the matrices 26 and 27 respectively:

$$
\mathscr{V}_{i}=\left(\begin{array}{c}
\left(\lambda_{2}+\tau_{2} \omega_{2}\right) L_{f}(t) \\
-\lambda_{2} L_{f}(t)+\left(\lambda_{3}+\tau_{3} \omega_{3}\right) P_{f}(t) \\
-\kappa \lambda_{3} P_{f}(t)-\delta_{1} F_{f f}(t)+\left[\lambda_{4}+\tau_{4} \omega_{4}\right] F_{f}(t) \\
-(1-\kappa) \lambda_{3} P_{f}(t)+\left[\left(\frac{F_{P}}{F_{f}+F_{p}}\right)+\tau_{5} \omega_{5}\right] M_{f}(t) \\
-\lambda_{4} F_{f}(t)+\left(\delta_{1}+\tau_{6} \omega_{6}(t)\right) F_{f f}(t) \\
-r F_{f f}(t)\left(1-\frac{E_{f}(t)}{A}\right) \phi S_{h}(t)+\left(\lambda_{2}+\tau_{2} \omega_{2}\right) L_{f}(t)
\end{array}\right)
$$

and

$$
\mathscr{F}_{i}=\left(\begin{array}{c}
\lambda_{1} E_{f}(t)+\frac{a \xi S_{h}(t) L_{f}(t)}{m+S_{h}(t)} \\
0 \\
0 \\
0 \\
0 \\
0
\end{array}\right)
$$

To obtain the next generation operator, we compute the Jacobian Matrices of $\mathscr{F}_{i}$ and $\mathscr{V}_{i}$ and solve it at the PFE with $E_{0}=\left(K_{h}\left(\alpha-\mu_{1}\right), 0,0,0,0,0,0,0\right)$ denoted by $\mathrm{F}$ and $\mathrm{V}$ respectively. Consequently, the basic offspring number of the system model 10 is obtained by determining the spectral radius of the matrix $F V^{-1}$ as given in the equation 28 :

$$
\mathscr{R}_{0}=\frac{a \xi K_{h}\left(\alpha-\mu_{1}\right)}{\left(m+K_{h}\left(\alpha-\mu_{1}\right)\right)\left(\lambda_{2}+\tau_{2} \omega_{2}\right)}
$$

In this study, the basic reproduction number measures the average number of new offspring produced by a fertilized fertile female in a completely susceptible host population at carrying capacity. Because the basic reproduction number $\mathscr{R}_{0}$ is less than one, the probability of FCM infestation in the susceptible host is decreasing.

\subsection{Stability Analysis}

The local stability of the equilibrium points of the model system 10 is investigated in this section.

\subsubsection{Local Stability Analysis}

To perform the local stability analysis of the model system 10, we begin by constructing the model system 10's Jacobian matrix and solving it at the equilibrium points. When the eigenvalues are positive real parts, the equilibrium points are unstable; however, when the eigenvalues are negative real parts, the equilibrium points are stable. The vector representation of system 10 is given in equation 29 :

$\frac{d y}{d t}=f(y)$

where:

$y=\left(S_{h}(t), E_{f}(t), L_{f}(t), P_{f}(t), F_{f}(t), M_{f}(t), F_{f f}(t), F_{p}(t)\right)$

Then, the function $y$ can be written in matrix form as shown in equation 30 :

$$
f(y)=\left(\begin{array}{c}
\alpha\left(1-\frac{S_{h}(t)}{K_{h}}\right) S_{h}(t)-\frac{\xi S_{h}(t) L_{f}(t)}{m+S_{h}(t)}-\mu_{1} S_{h}(t) \\
r F_{f f}(t)\left(1-\frac{E_{f}(t)}{A}\right) \phi S_{h}(t)-\left(\lambda_{1}+\tau_{1} \omega_{1}\right) E_{f}(t) \\
\lambda_{1} E_{f}(t)+\frac{a \xi S_{h}(t) L_{f}(t)}{m+S_{h}(t)}-\left(\lambda_{2}+\tau_{2} \omega_{2}\right) L_{f}(t) \\
\lambda_{2} L_{f}(t)-\left(\lambda_{3}+\tau_{3} \omega_{3}\right) P_{f}(t) \\
\kappa \lambda_{3} P_{f}(t)+\delta_{1} F_{f f}(t)-\left[\lambda_{4}+\tau_{4} \omega_{4}\right] F_{f}(t) \\
(1-\kappa) \lambda_{3} P_{f}(t)-\left[\left(\frac{F_{P}}{F_{f}+F_{p}}\right)+\tau_{5} \omega_{5}\right] M_{f}(t) \\
\lambda_{4} F_{f}(t)-\left(\delta_{1}+\tau_{6} \omega_{6}(t)\right) F_{f f}(t) \\
v-\mu F_{p}(t)
\end{array}\right)
$$

Clearly, the right hand side of equation 30 is continuous and locally Lipschitz, so uniqueness and local existence of the solution is guaranteed. By computing the Jacobian matrix of equation 30 yields equation 31 :

$$
\mathscr{J}_{f}=\left(\begin{array}{cccccccc}
x_{1} & 0 & x_{2} & 0 & 0 & 0 & 0 & 0 \\
x_{3} & x_{4} & 0 & 0 & 0 & 0 & x_{5} & 0 \\
x_{6} & x_{7} & x_{8} & 0 & 0 & 0 & 0 & 0 \\
0 & 0 & x_{9} & x_{10} & 0 & 0 & 0 & 0 \\
0 & 0 & 0 & x_{11} & x_{12} & 0 & x_{13} & 0 \\
0 & 0 & 0 & x_{14} & x_{15} & x_{16} & 0 & x_{17} \\
0 & 0 & 0 & 0 & x_{18} & 0 & x_{19} & 0 \\
0 & 0 & 0 & 0 & 0 & 0 & 0 & x_{20}
\end{array}\right)
$$


Where: $x_{1}=\alpha-\frac{2 S_{h}}{K_{h}}-\frac{\xi L_{f}\left(m+S_{h}\right)-\xi S_{h} L_{f}}{\left(m+S_{h}\right)^{2}}-\mu_{1}, x_{2}=-\frac{\xi S_{h}}{m+S_{h}}, x_{3}=r F_{f f}\left(1-\frac{E_{f}}{A}\right) \phi, x_{4}=-\frac{r F_{f f} \phi S_{h}}{A}-\left(\lambda_{1}+\tau_{1} \omega_{1}\right), x_{5}=r\left(1-\frac{E_{f}}{A}\right) \phi S_{h}$, $x_{6}=\frac{a m \xi L_{f}}{\left(m+S_{h}\right)^{2}}, x_{7}=\lambda_{1}, x_{8}=\frac{a \xi S_{h}}{m+S_{h}}-\left(\lambda_{2}+\tau_{2} \omega_{2}\right), x_{9}=\lambda_{2}, x_{10}=-\left(\lambda_{3}+\tau_{3} \omega_{3}\right), x_{11}=\kappa \lambda_{3}, x_{12}=-\left(\lambda_{4}+\tau_{4} \omega_{4}\right), x_{13}=\delta_{1}, x_{14}=(1-\kappa) \lambda_{3}$, $x_{15}=\frac{-F_{p} M_{f}}{\left(F_{f}+F_{p}\right)^{2}}, x_{16}=-\tau_{5} \omega_{5}, x_{17}=\frac{F_{f} M_{f}}{\left(F_{f}+F_{p}\right)^{2}}, x_{18}=\lambda_{4}, x_{19}=-\left(\delta_{1}+\tau_{6} \omega_{6}\right), x_{20}=-\mu$. The Jacobian matrix 31 is evaluated at both the pest free and coexistence equilibrium points, which are $E_{0}$ and $E_{2}$, respectively. If the eigenvalues are all positive, the equilibrium point is said to be unstable, but if all the eigenvalues are negative, the equilibrium point is said to be stable.

Theorem 2. The PFE point $E_{0}$ is locally asymptotically stable if $R_{0}<1$ and otherwise unstable

Proof. The local stability analysis of the model system 10 can be determined by solving the Jacobian matrix 31 at the pest free equilibrium points $\left(E_{0}=\left(K_{h}\left(\alpha-\mu_{1}\right), 0,0,0,0,0,0,0\right)\right.$, which yields equation 32 :

$$
\mathscr{J}_{f} \mid E_{0}=\left(\begin{array}{cccccccc}
x_{1} & 0 & x_{2} & 0 & 0 & 0 & 0 & 0 \\
0 & x_{4} & 0 & 0 & 0 & 0 & x_{5} & 0 \\
0 & x_{7} & x_{8} & 0 & 0 & 0 & 0 & 0 \\
0 & 0 & x_{9} & x_{10} & 0 & 0 & 0 & 0 \\
0 & 0 & 0 & x_{11} & x_{12} & 0 & x_{13} & 0 \\
0 & 0 & 0 & x_{14} & 0 & x_{16} & 0 & 0 \\
0 & 0 & 0 & 0 & x_{18} & 0 & x_{19} & 0 \\
0 & 0 & 0 & 0 & 0 & 0 & 0 & x_{20}
\end{array}\right)
$$

Where: $x_{1}=\mu_{1}-\alpha, x_{2}=-\frac{\xi K_{h}\left(\alpha-\mu_{1}\right)}{m+K_{h}\left(\alpha-\mu_{1}\right)}, x_{4}=-\left(\lambda_{1}+\tau_{1} \omega_{2}\right), x_{5}=r K_{h}\left(\alpha-\mu_{1}\right), x_{7}=\lambda_{1}, x_{8}=\frac{a \xi K_{h}\left(\alpha-\mu_{1}\right)}{m+K_{h}\left(\alpha-\mu_{1}\right)}-\left(\lambda_{2}+\tau_{2} \omega_{2}\right), x_{9}=\lambda_{2}$, $x_{10}=-\left(\lambda_{3}+\tau_{3} \omega_{3}\right), x_{11}=\kappa \lambda_{3}, x_{12}=-\left(\lambda_{4}+\tau_{4} \omega_{4}\right), x_{13}=\delta_{1}, x_{14}=(1-\kappa) \lambda_{3}, x_{15}=-\left[\tau_{3} \omega_{3}\right], x_{16}=-\tau_{5} \omega_{5}, x_{17}=-\left(\delta_{1}+\tau_{6} \omega_{6}\right)$. $x_{18}=\lambda_{4}, x_{19}=-\left(\delta_{1}+\tau_{6} \omega_{6}\right), x_{20}=-\mu$. The matrix 27 is solved using the Wolfram mathematica software, an extract of the solution is as given by the following equation: $s_{1}=x_{1}, s_{2}=x_{16}, s_{3}=x_{20}, s_{4}=s_{5}=s_{7}=s_{8}=\left[x_{10} x_{13} x_{18} x_{4} x_{8}-x_{10} x_{12} x_{19} x_{4} x_{8}-x_{11} x_{18} x_{5} x_{7} x_{9}+\ldots\right]$. Because all of the eigenvalues of the pest free equilibrium are negative, if $R_{0}$, the pest free equilibrium point is said to be stable, but if $R_{0}>1$, the equilibrium point is unstable. As a result, the system 10 is locally asymptotically stable around the pest-free equilibrium point $\left(E_{0}=\left(K h\left(\alpha-\mu_{1}\right), 0,0,0,0,0,0,0\right)\right.$

Theorem 3. The coexistence equilibrium point $E_{2}$ is locally asymptotically stable if $\mathbb{R}_{0}<1$ and otherwise unstable

Proof. The local stability analysis of the model system 10 can be established by solving the Jacobian matrix 31 at the coexistence equilibrium points $\left(E_{2}\right)$, using wolfram mathematica software the eigenvalues are found to be: $s_{1}=x_{16}, s_{2}=x_{20}, s_{3}=s_{4}=s_{5}=s_{6}=s_{7}=$ $s_{8}=\left[x_{10} x_{13} x_{18} x_{2} x_{4} x_{6}-x_{10} x_{12} x_{19} x_{2} x_{4} x_{6}-x_{10} x_{13} x_{18} x_{2} x_{3} x_{7}+x_{10} x_{12} x_{19} x_{2} x_{3} x_{7}-x_{1} x_{10} x_{13} x_{18} x_{4} x_{8}+x_{1} x_{10} x_{12} x_{19} x_{4} x_{8}+x_{1} x_{11} x_{18} x_{5} x_{7} x_{9}+\ldots\right]$. Since all the eigenvalues of the coexistence equilibrium points are negative, then the coexistence equilibrium point is a stable equilibrium point. The system 10 is therefore locally asymptotically stable around the the coexistence equilibrium points $E_{2}$.

\subsection{Global Stability Analysis}

Global stability should be used in a restricted sense for population models, that is, the definition should be limited to the feasible region. As a result, if a disturbance shifts the system's state to any other feasible state and the system is then left alone, the system's natural dynamics will move the state back into a small neighborhood of the equilibrium. If the disturbance resulted in the extinction of one species or the invasion of a new species, the system should be thought of mathematically and biologically as a new system. As a result, the new system's equilibrium points and stability will be examined [24].

\subsubsection{Global Stability of PFE}

To investigate the PFE's global asymptotic stability, one common approach is to build an appropriate Lyapunove function, as proposed by [49]. However, it is simpler to use the method developed by Catillo-Chevez et al. [19] and adopted by Bhunu and Mushayabasa [11]. As a result, we investigate the global stability of the system model 10 around the pest-free equilibrium point $\left(E_{0}\right)$. Assuming the system is cooperative on $\mathbb{R}_{+} 8$, the growth of any compartment has a positive impact on the growth of all other compartments. Using Catillo-Chevez et al . [19]'s theorem, we investigate the global asymptotic stability of the pest-free equilibrium by rewriting the system 10 in the form shown in equation 33 :

$$
\begin{aligned}
& \frac{d X}{d t}=F(X, Z) \\
& \frac{d Z}{d t}=G(X, Z), G(X, 0)=0
\end{aligned}
$$

Where: $X=\left(S_{h}, F_{p}\right) \in \mathbb{R}_{+}^{2}$ denotes noninfectious compartments and $Z=\left(E_{f}, L_{f}, P_{f}, F_{f}, M_{f}, F_{f f},\right) \in \mathbb{R}_{+}^{6}$ denotes the infectious pest compartments. $E_{0}=\left(X^{*}, 0\right)$ represents the pest free equilibrium of the system. If this points satisfies the following conditions: (i) for $\frac{d X}{d t}=F(X, 0)$, where $X^{*}$ is globally asymptotically stable, (ii) $\frac{d Z}{d t}=D_{z} G(X, 0) Z-G(X, Z), G(X, Z) \geq 0$ for all $(X, Z) \in \Omega$, then, we can conclude that $E_{0}$ is globally asymptotically stable if the following theorem 33 holds:

Theorem 4. The equilibrium point $E_{0}=\left(X^{*}, 0\right)$ of the system 33 is globally asymptotically stable if $\mathscr{R}_{0} \leq 1$ and the conditions ( $\left.i\right)$ and (ii) are satisfied. 
Proof. To prove this, we start by defining new variables and dividing the system 10 into sub systems $X=\left(S_{h}\right)$ and $Z=\left(E_{f}, L_{f}, P_{f}, F_{f}, M_{f}\right.$, $\left.F_{f f}\right)$. From equation 33, we have two vector valued functions $G(X, Z)$ and $F(X, Z)$ given by equations 34 and 35 :

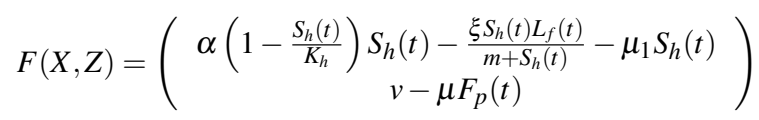

and

$G(X, Z)=\left(\begin{array}{c}r F_{f f}(t)\left(1-\frac{E_{f}(t)}{A}\right) S_{h}(t)-\left(\lambda_{1}+\tau_{1} \omega_{1}\right) E_{f}(t) \\ \lambda_{1} E_{f}(t)+\frac{a \xi S_{h}(t) L_{f}(t)}{m+S_{h}(t)}-\left(\lambda_{2}+\tau_{2} \omega_{2}\right) L_{f}(t) \\ \lambda_{2} L_{f}(t)-\left(\lambda_{3}+\tau_{3} \omega_{3}\right) P_{f}(t) \\ \kappa \lambda_{3} P_{f}(t)+\delta_{1} F_{f f}(t)-\left(\lambda_{4}+\tau_{4} \omega_{4}\right) F_{f}(t) \\ (1-\kappa) \lambda_{3} P_{f}(t)-\left[\left(\frac{F_{P}}{F_{f}+F_{p}}\right)+\tau_{5} \omega_{5}\right] M_{f}(t) \\ \lambda_{4} F_{f}(t)-\left(\delta_{1}+\tau_{6} \omega_{6}(t)\right) F_{f f}(t)\end{array}\right)$

Now, let consider the reduced system, $\frac{d X}{d t}=F(X, 0)$ from condition (i) yields:

$$
\begin{aligned}
\frac{d S_{h}}{d t} & =\alpha\left(1-\frac{S_{h}(t)}{K_{h}}\right) S_{h}(t)-\mu S_{h}(t) \\
\frac{d F_{p}}{d t} & =v-\mu F_{p}(t)
\end{aligned}
$$

We note that this is asymptomatic dynamics system, independence of the initial conditions in $\mathscr{D}$, therefore the convergence of the solutions of the reduced system 32 is global in $\mathscr{D}$. We then compute:

$G(X, Z)=D_{z} G\left(X^{*}, 0\right)-\hat{G}(X, Z)$

and show that

$\hat{G}(X, Z) \geq 0$

Now, we let $B=D_{z} G\left(X^{*}, 0\right)$, which is the Jacobian of $\hat{G}(X, Z)$ taken in $\left(E_{f}, L_{f}, P_{f}, F_{f}, M_{f}, F_{f f}\right)$ and evaluated at $\left(X^{*}, 0\right)$. Such that the matrix $B$ is given by the equation 37 :

$B=\left(\begin{array}{cccccc}x_{1} & 0 & 0 & 0 & 0 & x_{2} \\ x_{3} & x_{4} & 0 & 0 & 0 & 0 \\ 0 & x_{5} & x_{6} & 0 & 0 & 0 \\ 0 & 0 & x_{7} & x_{8} & 0 & x_{9} \\ 0 & 0 & x_{11} & 0 & x_{12} & 0 \\ 0 & 0 & 0 & 0 & 0 & x_{13}\end{array}\right)$

Where: $x_{1}=-\left(\lambda_{1}+\tau_{1} \omega_{1}\right), x_{2}=-r K_{h}\left(\alpha-\mu_{1}\right), x_{3}=\lambda_{1}, x_{4}=\frac{a \xi K_{h}\left(\alpha-\mu_{1}\right)}{m+K_{h}\left(\alpha-\mu_{1}\right)}-\left(\lambda_{2}+\tau_{2} \omega_{2}\right), x_{5}=\lambda_{2}, x_{6}=-\left(\lambda_{3}+\tau_{3} \omega_{3}\right), x_{7}=\kappa \lambda_{3}$, $x_{8}=-\tau_{4} \omega_{4}, x_{9}=\delta_{1}, x_{10}=\delta_{2}, x_{11}=(1-\kappa) \lambda_{3}, x_{12}=-\left[\tau_{5} \omega_{5}, x_{13}=-\left(\delta_{1}+\tau_{6} \omega_{6}\right), x_{14}=\lambda_{4}, x_{15}=-\left(\delta_{2}+\tau_{7} \omega_{7}\right)\right.$.

The value for $\hat{G}(X, Z)$ is given by equation 38 :

$\hat{G}(X, Z)=\left(\begin{array}{c}r F_{f f}(t)\left(1-\frac{E_{f}(t)}{A}\right)\left(K_{h}\left(\alpha-\mu_{1}\right)-S_{h}(t)\right. \\ a \xi L_{f}(t)\left(\frac{\left(K_{h}\left(\alpha-\mu_{1}\right)\right.}{m+\left(K_{h}\left(\alpha-\mu_{1}\right)\right.}-\frac{S_{h}}{m+S_{h}}\right) \\ 0 \\ 0 \\ 0 \\ 0\end{array}\right)$

Since $K_{h} \geq S_{h}$, it is obvious that $\hat{G}(X, Z) \geq 0$ for all $(X, Z) \in \mathscr{D}$, the pest-free equilibrium will be globally asymptotically stable. We can also see that matrix $B$ is an M-matrix because all of its off-diagonal elements are non-negative. As a result, this demonstrates that PFE is globally asymptotically stable.

As a result of these findings, the pest-free equilibrium will be globally asymptotically stable. However, whenever the FCM diapausing, the PFE may not be globally asymptotically stable, which is not true from the values of $\hat{G}(X, Z) \geq 0$ everywhere in $\mathscr{D}$, implying the existence of multiple coexistence equilibria. 


\subsubsection{Global Stability Analysis of Coexistence Equilibrium}

Theorem 5. In the model of host-pest interactions, sufficient conditions for global stability are (i) a feasible coexistence equilibrium point and (ii) the equilibrium is a local asymptomatic stable solution.

Proof. Global stability of coexistence equilibrium points can be constructed using a suitable Lyapunov function an approach adopted by [31;47]. In this approach Lyapunov function is constructed basing on equation 39:

$$
L=\sum a_{i}\left(x_{i}-x_{i}^{* \&} \ln x_{i}\right)
$$

Where $a_{i}$ is the constant selected such that $a_{i}>0, x_{i}$ is the population of the ith compartment and $x_{i}^{* \&}$ is the coexistence equilibrium point. Therefore, consider the Lyapunove function in equation 40 :

$$
\begin{aligned}
L= & a_{1}\left(S_{h}-S_{h}^{* \&} \ln S_{h}\right)+a_{2}\left(E_{f}-E_{f}^{* \&} \ln E_{f}\right)+a_{3}\left(L_{f}-L_{f}^{* \&} \ln L_{f}\right)+a_{4}\left(P_{f}-P_{f}^{* \&} \ln P_{f}\right) \\
& +a_{5}\left(F_{f}-F_{f}^{* \&} \ln F_{f}\right)+a_{6}\left(M_{f}-M_{f}^{* \&} \ln M_{f}\right)+a_{7}\left(F_{f f}-F_{f f}^{* \&} \ln F_{f f}\right)+a_{8}\left(F_{p}-F_{p}^{* \&} \ln F_{p}\right)
\end{aligned}
$$

Differentiating 40 with respect to time yields equation 41 :

$$
\begin{aligned}
\frac{d L}{d t}= & a_{1}\left(1-\frac{S_{h}^{* \&}}{S_{h}}\right) \frac{d S_{h}}{d t}+a_{2}\left(1-\frac{E_{f}^{* \&}}{E_{f}}\right) \frac{d E_{f}}{d t}+a_{3}\left(1-\frac{L_{f}^{* \&}}{L_{f}}\right) \frac{d L_{f}}{d t}+a_{4}\left(1-\frac{P_{f}^{* \&}}{P_{f}}\right) \frac{d P_{f}}{d t} \\
& +a_{5}\left(1-\frac{F_{f}^{* \&}}{F_{f}}\right) \frac{d F_{f}}{d t}+a_{6}\left(1-\frac{M_{f}^{* \&}}{M_{f}}\right) \frac{d M_{f}}{d t}+a_{7}\left(1-\frac{F_{f f}^{* \&}}{F_{f f}}\right) \frac{d F_{f f}}{d t}+a_{8}\left(1-\frac{F_{p}^{* \&}}{F_{p}}\right) \frac{d F_{p}}{d t} \\
& =a_{1}\left(1-\frac{S_{h}^{*}}{S_{h}}\right)\left[\alpha\left(1-\frac{S_{h}(t)}{K_{h}}\right) S_{h}(t)-\frac{\xi S_{h}(t) L_{f}(t)}{m+S_{h}(t)}-\mu_{1} S_{h}(t)\right] \\
& +a_{2}\left(1-\frac{E_{f}^{* \&}}{E_{f}}\right)\left[r F_{f f}(t)\left(1-\frac{E_{f}(t)}{A}\right) S_{h}(t)-\left(\lambda_{1}+\tau_{1} \omega_{1}\right) E_{f}(t)\right] \\
& +a_{3}\left(1-\frac{L_{f}^{* \&}}{L_{f}}\right)\left[\lambda_{1} E_{f}(t)+\frac{a \xi S_{h}(t) L_{f}(t)}{m+S_{h}(t)}-\left(\lambda_{2}+\tau_{2} \omega_{2}\right) L_{f}(t)\right] \\
& +a_{4}\left(1-\frac{P_{f}^{* \&}}{P_{f}}\right)\left[\lambda_{2} L_{f}(t)-\left(\lambda_{3}+\tau_{3} \omega_{3}\right) P_{f}(t)\right] \\
& +a_{5}\left(1-\frac{F_{f}^{* \&}}{F_{f}}\right)\left[\kappa \lambda_{3} P_{f}(t)+\delta_{1} F_{f f}(t)-\left[\lambda_{4}+\tau_{4} \omega_{4}\right] F_{f}(t)\right] \\
& +a_{6}\left(1-\frac{M_{f}^{* \&}}{M_{f}}\right)\left[(1-\kappa) \lambda_{3} P_{f}(t)-\left[\left(\frac{F_{P}}{F_{f}+F_{p}}\right)+\tau_{5} \omega_{5}\right] M_{f}(t)\right] \\
& +a_{7}\left(1-\frac{F_{f f}^{* \&}}{F_{f f}}\right)\left[\lambda_{4} F_{f}(t)-\left(\delta_{1}+\tau_{6} \omega_{6}(t)\right) F_{f f}(t)\right] \\
& \left(1-\frac{F_{p}^{* \&}}{F_{p}}\right)\left[v-\mu F_{p}(t)\right]
\end{aligned}
$$

Where: $S_{h}(t)=S_{h}^{* \&}, E_{f}(t)=E_{f}^{* \&}, L_{f}(t)=L_{f}^{* \&}, P_{f}(t)=P_{f}^{* \&}, F_{f}(t)=F_{f}^{* \&}, M_{f}(t)=M_{f}^{* \&}, F_{f f}(t)=F_{f f}^{* \&}, F_{p}(t)=F_{p}^{* \&}$.

Following the approach of McClusky [57], it can easily be seen that equation 37 is non-positive. Hence $\frac{d L}{d t} \leq 0 \forall S_{h}, E_{f}, L_{f}$ $, P_{f}, F_{f}, M_{f}, F_{f f}>0$ and is zero when $S_{h}(t)=S_{h}^{* \&}, E_{f}(t)=E_{f}^{* \&}, L_{f}(t)=L_{f}^{* \&}, P_{f}(t)=P_{f}^{* \&}, F_{f}(t)=F_{f}^{* \&}, M_{f}(t)=M_{f}^{* \&}, F_{f f}(t)=F_{f f}^{* \&}$. Therefore, the largest invariant set in $\left\{\left(S_{h}^{* \&}, E_{f}^{* \&}, L_{f}^{* \&}, P_{f}^{* \&}, F_{f}^{* \&}, M_{f}^{* \&}, F_{f f}^{* \&}, F_{p}^{* \&}\right) \in \mathscr{D}\right\}$ such that $\frac{d L}{d t}=0$ is the singleton $\left\{E_{2}\right\}$ which is the coexistence equilibrium point. According to the invariant principle put forward by Lasalles [30], $E_{2}$ is globally asymptotically stable in $\mathscr{D}$, if $R_{0} \leq 1$ the interior of $\mathscr{D}$, otherwise unstable.

This result implies that the elimination of FCM is possible regardless of the initial sizes of the model's FCM sub populations, as long as the threshold parameter $R_{0}$ is less than unity. As a result of the Lyapunov-LaSella invariance principle, system model 10 is uniformly persistent.

\subsection{Numerical Simulation}

The impact of pheromone traps as a control measure on the susceptible host and FCM is numerically simulated using MATLAB software using the parameter values in Tables 1 . The role and impact of pheromone trap on the susceptible host is illustrated in graphically. Figure 4 shows a graph of pheromone concentration against time within a period of 100 days, with the initial concentration set at $0 \mathrm{mg}$, the concentration is then increased at the rate of $10 \mathrm{mg}$ per day that is $v=10$. From the Figure its observed that the concentration of pheromone increases logistically with time and reaches a maximum value of about 110 after 51 days. This maximum value of the pheromone is considered to effective concentration that offers the effective control against FCM. When different concentration is used the pheromone attains the maximum value faster. 


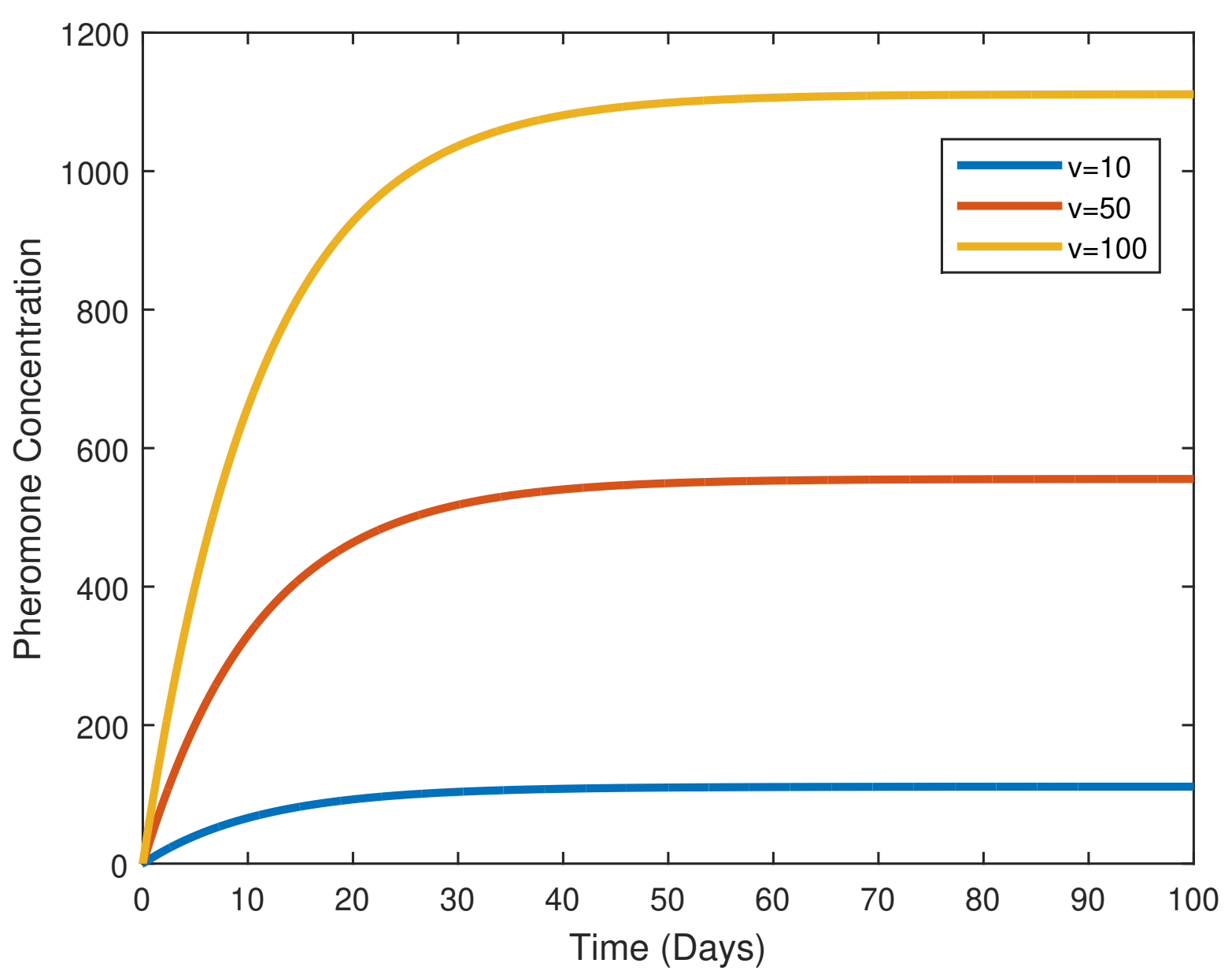

Figure 4: A plot of Pheromone Concentration against Time (Days)

Figure 5 shows a graph of susceptible host against pheromone concentration, with the initial concentration of the pheromone in the field set at $0 \mathrm{ngm}^{-3}$ and the pheromone concentration was increased at the rate of $5 \mathrm{mg}, 10 \mathrm{mg}, 50 \mathrm{mg}$, and 100mg per day. From the Figure it is observed that all the release rates have a controlling effect of FCM as all the the release rates enables the susceptible host to attain its carrying capacity at $K_{h}=1000$ that is the susceptible host population increases with the increase of the pheromone concentration up to the carrying capacity of the susceptible host. Therefore, for cost effect control measure its important to determine the minimum effective pheromone concentration to be released in the field as higher concentration may lead to FCM Confusion but not control. 


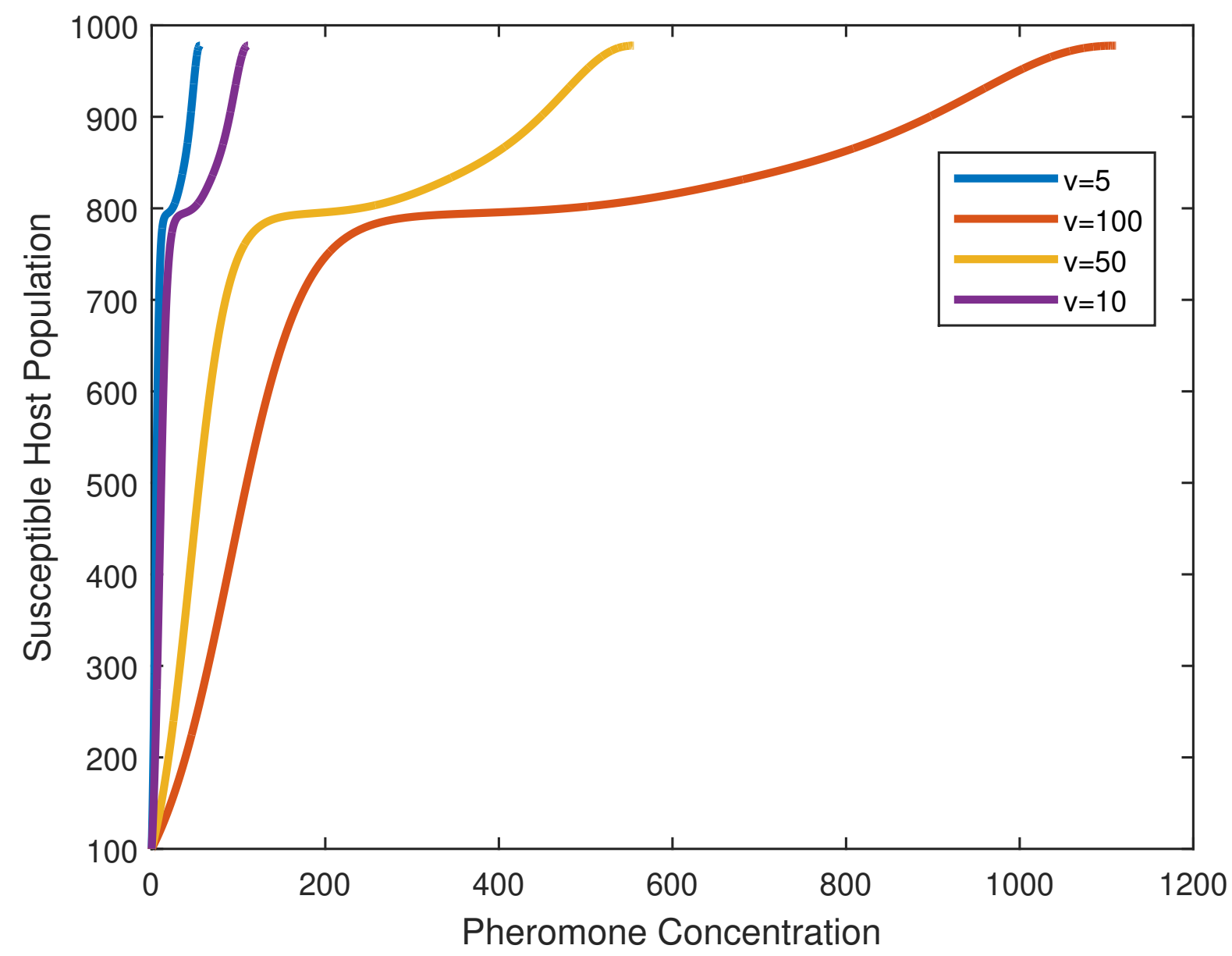

Figure 5: A plot of the Susceptible host against Pheromone Concentration

Figure 6 shows a plot of total FCM population against pheromone concentration when the initial FCM population is set at 600 FCM within a period of 100 days and $v=5, v=10, v=50$ and $v=100$. From the Figure the FCM population rises sharply to maximum value of about 5015 and then sharply drops to near zero. This further illustrates the effectiveness of the pheromone trap as a control strategies, thus a minimum threshold value that can offer an effective control strategy and cost effective should be established. 


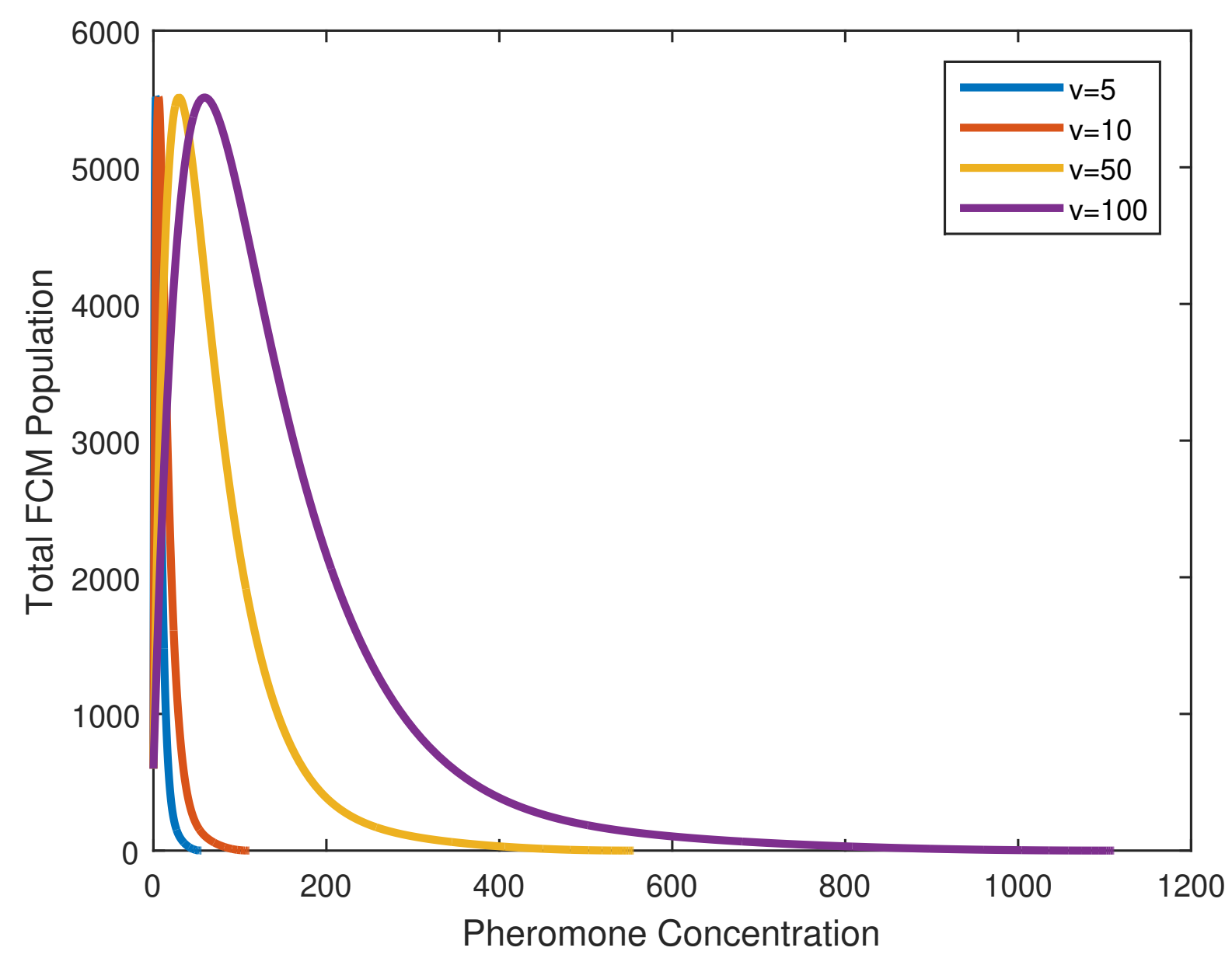

Figure 6: A plot of Total FCM Population against Pheromone Concentration

Figure 7 illustrates a plot of fertile male population against pheromone concentration under different release rate of pheromone concentration per day. From the graph the number of fertile male decreases sharply with the increase in pheromone concentration to near zero. This indicates the effectiveness of pheromone traps on fertile male because once they are attracted to the pheromone traps they are killed hence the sharp decrease in their population in the presence of pheromone. 


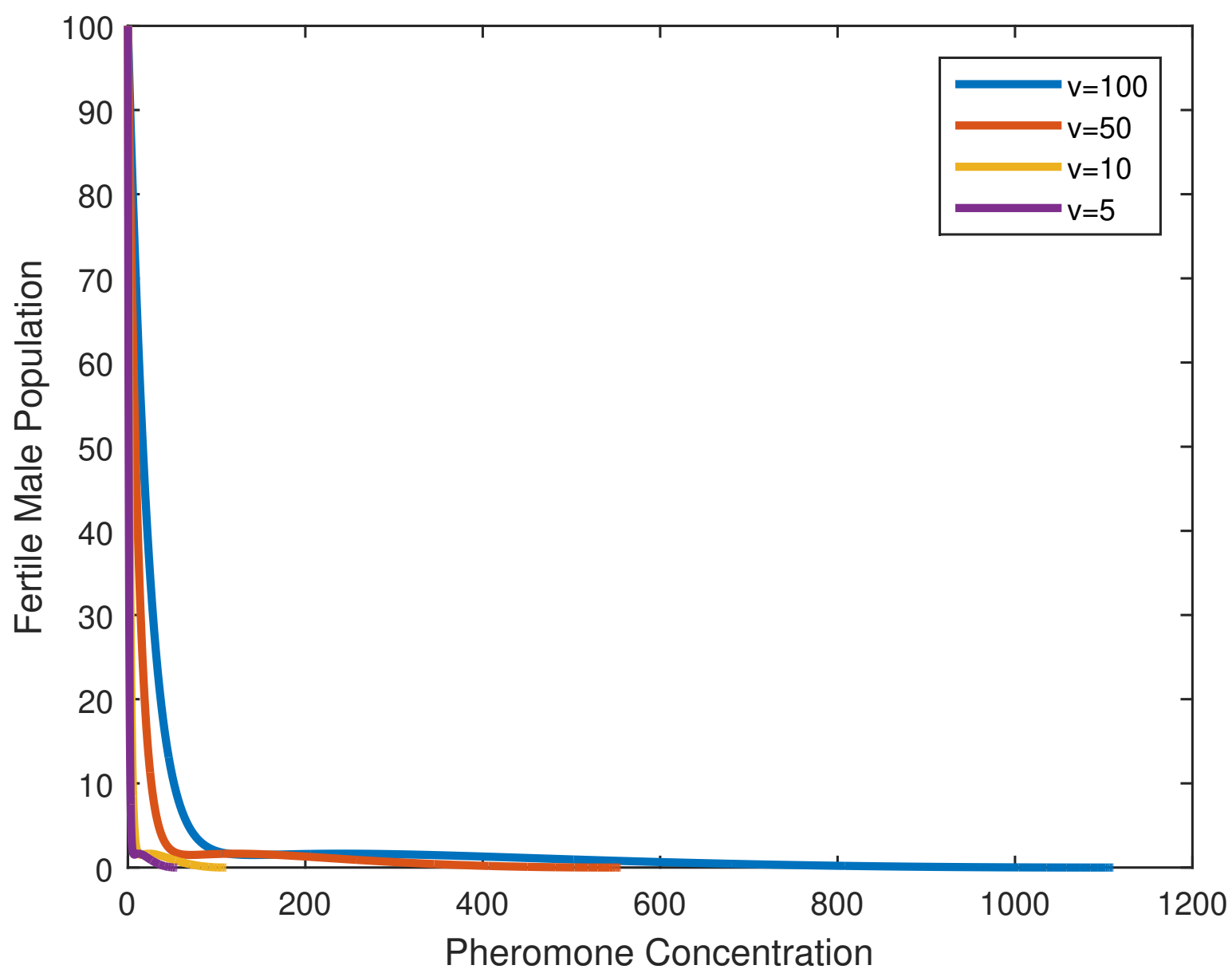

Figure 7: A plot of Fertile Male Population against Pheromone Concentration

The impact of FCM on the susceptible host in the absence of pheromone the is illustrated graphically in figures 8 and 9 . A graph of susceptible host against time in the absence of pheromone traps for a period of 30 days is illustrated in Figure 8 . From the graph the population of the susceptible grows from the initial population of 100 logistically and level off at 428 . Since the carrying capacity $K_{h}$ of the susceptible host is set at 1000, the presence of the FCM pest clearly makes the susceptible host to grow below its carrying capacity. When the initial population is increased to 500 the susceptible host population drops exponentially and again levels off at 428 , this is due to the effect of FCM on the population of the susceptible host. 


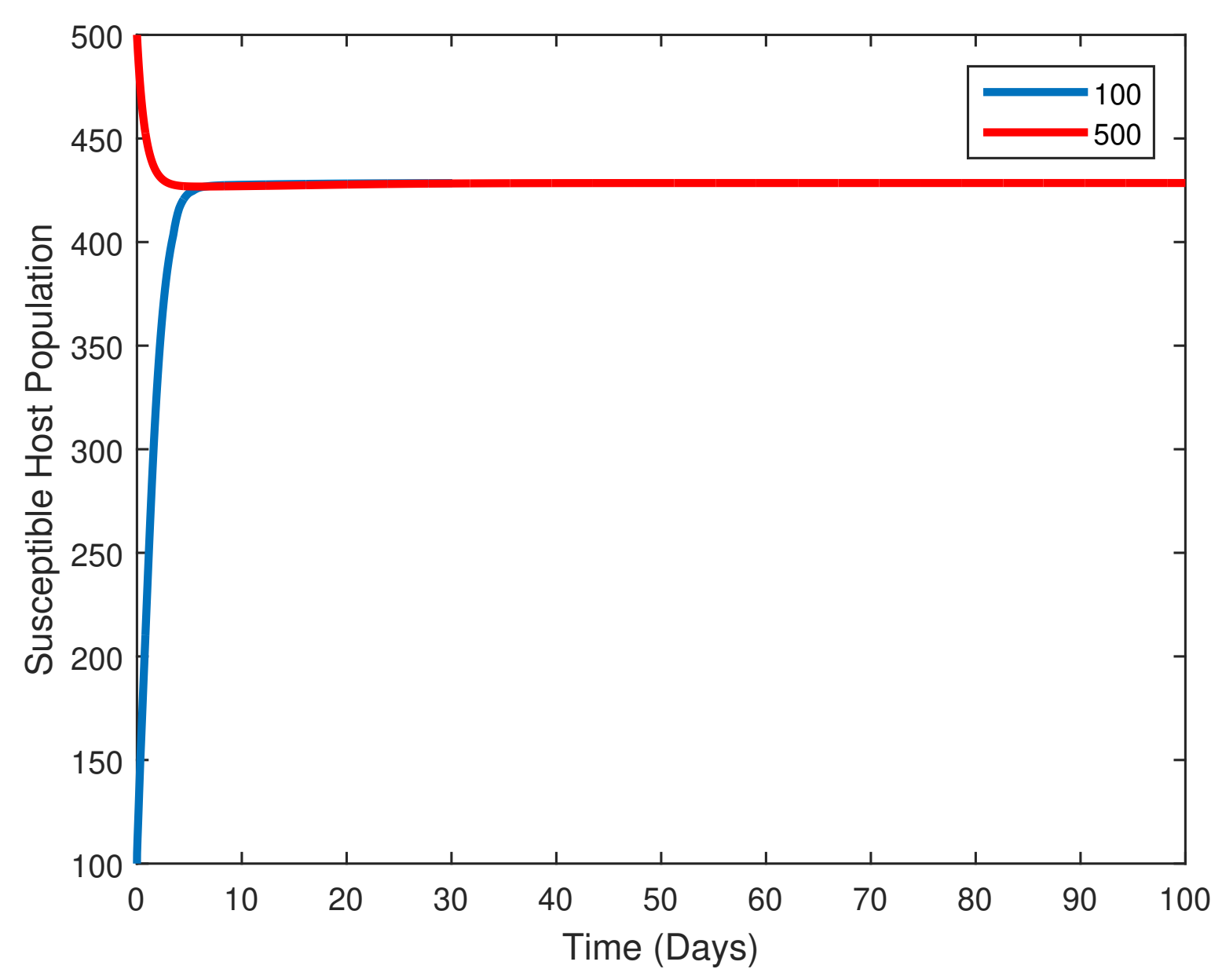

Figure 8: A plot of Susceptible Host against Time in the absence of Pheromone Traps

Figure 9 illustrates a graph of FCM total population with time in the absence of pheromone in a period of 30 days. From the graph its observed that the FCM population first sharply increase from the initial population of 1500 to a maximum population of 372099 , then starts to drops gradually and levels off at a population of 4272 after 30 day. If the initial population of FCM in the field is reduced to about 100, the population of FCM is observed to rise to a maximum value of 30575 , then drops gradually and levels at the minimum value of 1757 . The first increase is due to the availability of food for the FCM pest as time goes by the available number of susceptible host starts to decline leading to the decline of the FCM population in the field. The population of the FCM does not drop to zero because the susceptible host is continuously being introduced in the field. 


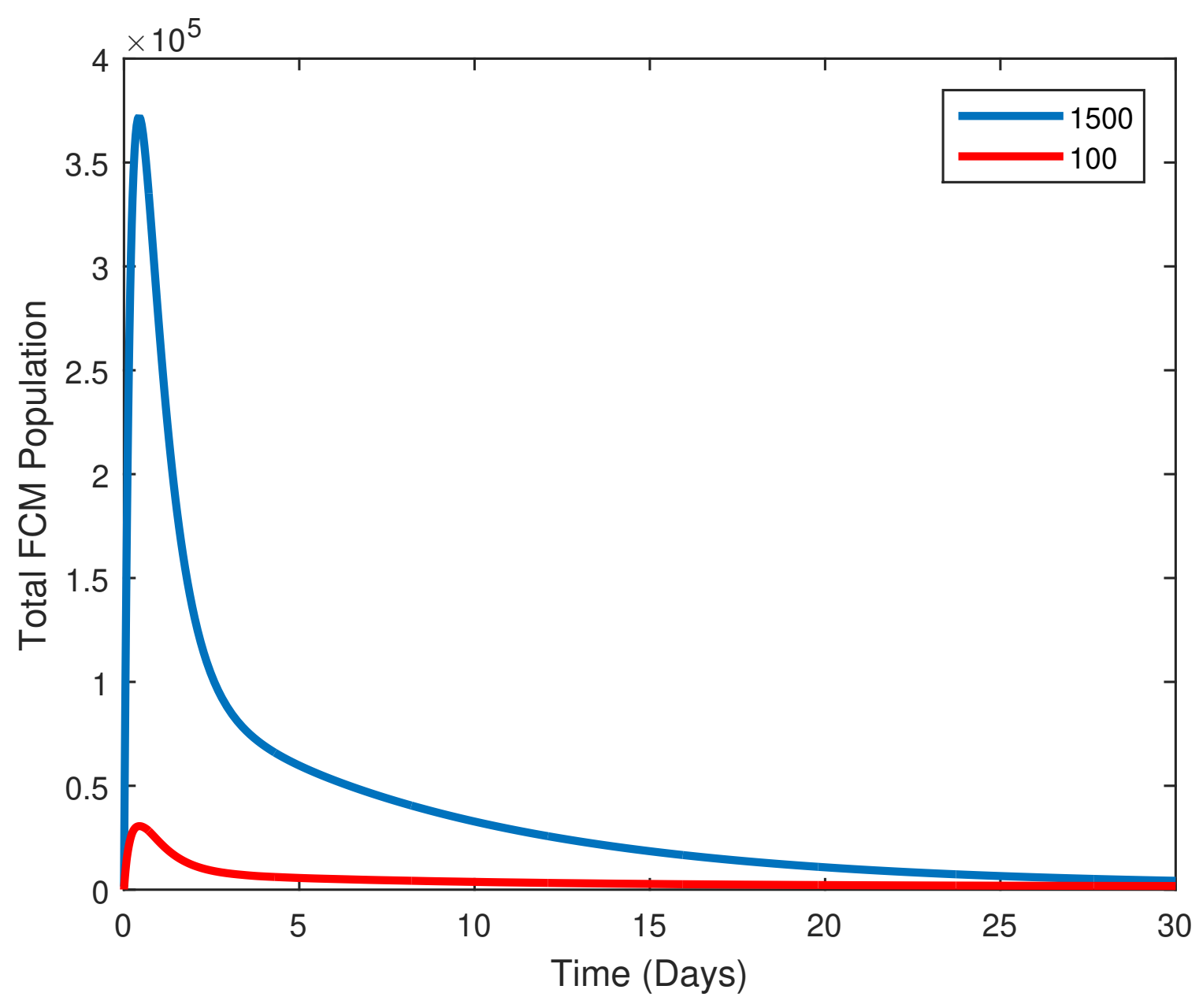

Figure 9: A plot of Total FCM Population against Time without Pheromone Traps

\section{Conclusions}

In this paper, deterministic model of FCM control using pheromone traps is developed and carefully analysed to bring insight into its dynamical features of FCM control measures. The model is considered to be biologically and mathematically well posed. Using next generation matrix method the basic reproduction number is determined. The model was analysed qualitatively and the local and global stability of their associated equilibria. The dynamics of the FCM infestation on the susceptible host population showed that in the absence of any control measures the population of FCM would increase, leading to decrease of susceptible host population. Numerical simulation also shows that pheromone traps offers an effective control measure of FCM. From the numerical simulation it is also observed that the presence of FCM in a farm greatly affects the population of susceptible host, hence there is a need to adopt control measure such as pheromone traps to effectively increase farm produce.

\subsection{Recommendations}

In this study, we have only concentrated on the FCM interaction with the susceptible host with pheromone traps as the only control measure. Therefore, more control measures such as sterile insect release, biological control can be considered in the model in order to draw clear comparison on control strategy. There should be more enlightenment campaign on the economic effects of FCM to crops and identification method of FCM on crops by the farmers.

\subsection{Conflicts of interest}

There are no conflicts to declare.

\section{Acknowledgments}

We thanks the administrative staff in the department of physical science of Chuka University for their hospitality and assistance in matters related to our research work.

\section{References}

[1] W. C. Agosta, Chemical Communication: the Language of Pheromones. Henry Holt and Company, (1992). 
[2] H. T. Alemneh, O. D. Makinde, D. M. Theuri, Mathematical modelling of msv pathogen interaction with pest invasion on maize plant, Global Journal of Pure and Applied Mathematics, 15(1):55-79 (2019).

[3] R. Anderson, R. May, Population Biology of Infectious Diseases: Part I. Nature, 280:361-7.(1979).

[4] M.R.Anguelov, C.Dufourd, Y.Dumont, (2016), Mathematical Model for Pest-Insect Control using Mating Disruption and Trapping. Applied Mathematical Modelling. (2016).

[5] H. J. Barclay, G. E. Haniotakis, . Combining Pheromone-Baited and Food- Baited Traps for Insect Pest Control: Effects of Developmental Period. Population Ecology, 33(2):269-285 (1991).

[6] H. J. Barclay, R. Steacy, W. Enkerlin, P. van den Driessche, Modeling Diffusive Movement of Sterile Insects Released along Aerial Flight Lines International Journal of Pest Management, 62(3):228-244.(2016).

[7] H. J. Barclay, P. Van Den Driessche, A sterile Release Model for Control of a Pest with two Life Stages under Predation. The Rocky Mountain Journal of Mathematics, pages 847-855.(1990).

[8] H.Bestmann, J. Erler, O. Vostrowsky, Determination of Diel Periodicity of Sex Pheromone Release in Three Species of Lepidoptera by 'Closed-LoopStripping'. Experientia, 44(9) (1988):797-799

[9] Bhattacharyya, R. and Mukhopadhyay, B. (2014). Mathematical study of a pest control model incorporating sterile insect technique. Natural Resource Modeling, 27(1):61- 79.

[10] T.Blomefield, Economic Importance of False Codling Moth, Cryptophlebia leucotreta, and Codling Moth, Cydia Pomonella, on Peaches, Nectarines and Plums. Phytophylactica, 21(4):435-436.(1989).

[11] C.Bhunu, S. Mushayabasa, Modelling the transmission dynamics of pox-like infections. International Journal of Applied Mathematics.(2011).

[12] L. Boardman, T.G. Grout, J.S. Terblanche, . False codling moth thaumatotibia leucotreta (lepidoptera, tortricidae) larvae are chill-susceptible Insect Science, 19(3):315-328.(2012)

[13] J. H. Borden, Use of Semiochemicals to Manage Coniferous Tree Pests in Western Canada. In Behaviour Modifying Chemicals for Insect Management: Application of Pheromones and other Attractants, (1990).

[14] E. Boissard, P. Degond, S. Motsch, (2011). Trail Formation Based on Directed Pheromone Deposition. Hal.

[15] J. A.Byers, Simulation of Mating Disruption and Mass Trapping with Competitive Attraction and Camouflage. Environmental Entomology, 36(6):1328-1338. (2014).

[16] R. Cardé, Using pheromones to disrupt mating of moth pests. Perspectives in ecological theory and integrated pest management. Cambridge University Press, Cambridge, (2007). pages 122-169.

[17] R. T. Cardé, A. K. Minks, Control of Moth Pests by Mating Disruption: Successes and Constraints. Annual Review of Entomology, 40(1), (1995):559-585.

[18] R. T. Carde, Principles of Mating Disruption. Behavior-Modifying Chemicals for Pest Management: Applications of Pheromones and Other Attractants. Marcel Dekker, New York,(1990), pages 47-71.

[19] C. Castillo-Chavez, B. Song, Dynamical models of tuberculosis and their applications. Mathematical Biosciences and Engineering, 1(2):361.(2004).

[20] D. Chouinard,G.Vanoosthuyse,F.Pelletier, F.Bellerose, S.Bourgeois, P. Dominique, A Phenology Model for Codling Moth Management in Quebec Apple Orchards. Acta Horticulturae, 1068(5):51-56.(2015).

[21] G. Gianni, S. Pasquali, S. Parisi, S. Winter, Modelling the Potential Distribution of Bemisia Tabaciin Europe in Light of the Climate Change scenario. Pest Management science, 70:1611-1623.(2014).

[22] T. M. Gilligan, M. E. Epstein, K. M. Hoffman, Discovery of false codling moth, Thaumatotibia leucotreta (Meyrick), in California (Lepidoptera: Tortricidae). Proceedings of the Entomological Society of Washington, 113(4),(2011), 426-435.

[23] B.Goh, Global stability in two species interactions. Journal of Mathematical Biology, 3(3):313-318.(1976).

[24] P.Howse, J. Stevens, O.T. Jones, Insect Pheromones and their use in Pest Management. Springer Science and Business Media (2013).

[25] . H.Hofmeyr, M. Hofmeyr, M. Lee, H. Kong, M. Holtzhausen, Assessment of a Cold Treatment for the Disinfestations of Export Citrus from False Codling Moth, Thaumatotibia leucotreta (Lepidoptera: Tortricidae): Report to the People's Republic of China. Citrus Research International, http://www. citrusres. com/sites/default/files/documents/FCM\% 20cold\% 20disinfestation\% 20study\% 20f or\% 20Korea, 201998.(1998).

[26] FPEAK, Protocols for the Management of the False Codling Moth (Thaumatotibia Leucotreta) in Roses in Kenya. Kenya Flower Council Technical Committee, Kenya Plant Health Inspectorate Service (KEPHIS), Fresh Produce Exporters Association of Kenya (FPEAK), Kenya Agricultural and Livestock Research Organization (KALRO) and the Europe-Africa-Caribbean-Pacific Liaison Committee (COLEACP) in the scope of its NExT Kenya programme, (2021). https://fpeak.org/wp-content/uploads/2021/05/FCM-Manual.pdf

[27] Y.Ikemoto, Y. Ishikawa, T. Miura, H. Asama, A mathematical model for caste differentiation in termite colonies (isoptera) by hormonal and pheromonal regulations. Sociobiology, 54(3), 841. (2009).

[28] D. Kalajdzievska, M.Y. Li, Modeling the Effects of Carriers on the Transmissions Dynamics of Infectious Diseases. PhD thesis, University of Alberta,(2007).

[29] U. T. Koch, W. Lüder, U.Andrick, R. T. Staten, R. T. Cardé, Measurement by electroantennogram of airborne pheromone in cotton treated for mating disruption of Pectinophora gossypiella following removal of pheromone dispensers. Entomologia experimentalis et applicata, 130(1), 1-9. (2009).

[30] J. P. La Salle, An invariance principle in the theory of stability. Space Flight and Guidance Theory,(1966).

[31] X.Liu, B. and Dai, Threshold dynamics of a delayed predator-prey model with impulse via the basic reproduction number. Advances in Difference Equations, 2018(1):454.(2018).

[32] A. Korobeinikov, G. C. Wake, Lyapunov functions and global stability for sir, sirs, and sis epidemiological models. Applied Mathematics Letters, 15(8), (2002):955-960.

[33] R. M.May, R. M. Anderson, Regulation and Stability of Host-Parasite Population Interactions: II. Destabilizing Processes. The Journal of Animal Ecology, (1978) pages 249-267.

[34] M.Mkiga, S. Mohamed, H. du Plessis, F.Khamis, S. Ekesi, Field and Laboratory Performance of False Codling Moth, Thaumatotibia Leucotreta (Lepidoptera: Troticidae) on Orange and Selected Vegetables. Insects, 10(3),(2019):63.

[35] L. L. Mondaca, N. Da-Costa, A. Protasov, S. Ben-Yehuda, A. Peisahovich, Z Mendel, D. Ment, . Activity of metarhizium brunneum and beauveria bassiana against early developmental stages of the false codling moth thaumatotibia leucotreta. Journal of Invertebrate Pathology, 170, (2020):107312.

[36] S.Moore, Moths and Butterflies: False Codling Moth. Citrus Research International IPM Production Guidelines, 3 (2012)(Part 9.4).

[37] D. K. Mueller, (1995). Pheromones Chapter 25 in Moreland, D. (Ed.) Handbook of Pest Control, 8th Edition. Mallis Handbook and Technical Training Company, (1995).

[38] J.Murray, J. Mathematical Biology (eds Antman, SS, Marsden, JE, Sirovich, L. and Wiggins, S.) , (2002).175-256. 126

[39] M.Okongo, Modeling HIV-AIDS Co-Infections with Malaria and Tuberculosis in the Presence of Antiretroviral Treatment and Counseling. PhD thesis, Kenya: Chuka University, (2016).

[40] S. Pasquali,C. Soresina, G.Gilioli, G. The effects of fecundity, mortality and distribution of the initial condition in phenological models. Ecological modelling, 402, (2019):45-58.

[41] R. Peshin, A. K. Dhawan, Integrated Pest Management: Volume 1: Innovation Development Process, volume 1. Springer Science and Business Media , (2009).

[42] L. Potgieter, A mathematical Model for the Control of Eldana Saccharina Walker using the Sterile Insect Technique. PhD thesis, Stellenbosch: Stellenbosch University ,(2013a).

[43] S.Savary, P.S. Teng, L. Willocquet, F. W. Nutter Jr, Quantification and Modeling of Crop Losses: A Review of Purposes. Annu. Rev. Phytopathol, 44, (2006).:89-112.

[44] R.Sergio, The Optimal Release of Sterile Males in Pest Management. All Graduate Plan B and other Reports, 408. 128,(2014),

[45] J.Stibick, S. Bloem, J.Carpenter, S. Ellis, T. Gilligan, New Pest Pesponse Guidelines: False Codling Moth Thaumatotibia leucotreta. Technical report, USDA- APHIS-PPQ-Emergency and Domestic Programs, Riverdale, Maryland, (2008).

[46] Z. Tazerouni, A. Talebi, M. Rezaei, Functional response of parasitoids: Its impact on biological control. Parasitoids: Biology, Behavior, and Ecology. Nova Science Publishers Inc., New York, (2019)..

[47] M.B. Thomas and A.J. Willis, Biocontrol - risky but necessary, Trends Ecol. Evol. 13 (1998), pp. $325-329$.

[48] R.Ullah, G. Zaman, S. Islam, Stability analysis of a general sir epidemic model. VFAST Transactions on Mathematics, 1(1)(2013):57-61. 
[49] M.Rafikov, E. de Holanda Limeira, Mathematical modelling of the biological pest control of the sugarcane borer. International Journal of Computer Mathematics, 89(3),(2012), 390-401.

[50] P.Van den Driessche, J.Watmough, Reproduction numbers and sub-threshold endemic equilibria for compartmental models of disease transmission. Mathematical biosciences, 180(1-2)(2002):29-48.

[51] Z. Varga, Applications of mathematical systems theory in population biology, Period. Math. Hungar. 56 (1) (2008), pp. $157-168$.

[52] R. C. Venette, E. E. Davis, M. DaCosta, H. Heisler, M. Larson, Mini Risk Assessment False Codling Moth, Thaumatotibia (Cryptophlebia) leucotreta (Meyrick)(Lepidoptera: Tortricidae). University of Minnesota, Department of Entomology, CAPS PRA,(2003). pages 1-30.

[53] S.Verreynne, Fruit size and crop load prediction for citrus. SA Fruit Journal, 8(5), (2009):63-67.

[54] B. P. Wilson, L. K. Alan, F. C. Eduardo, Modeling Codling Moth (Lepidoptera: Tortricidae) Phenology and Predicting Egg Hatch in Apple Orchards of the Maule Region, Chile. Chilean Journal of Agricultural Research, (2015).

[55] P.Witzgall, P. Kirsch, A. Cork, . Sex Pheromones and their Impact on Pest Management. Journal of Chemical Ecology, 36(1),(2010):80-100.

[56] C. C. McCluskey, Global stability for an SIR epidemic model with delay and nonlinear incidence. Nonlinear Analysis: Real World Applications, 11(4),(2010), 3106-3109. 